This item is the archived peer-reviewed author-version of:

Enhancement of plasma generation in catalyst pores with different shapes

\title{
Reference:
}

Zhang Yu-Ru, Neyts Erik, Bogaerts Annemie.- Enhancement of plasma generation in catalyst pores w ith different shapes

Plasma sources science and technology / Institute of Physics [Londen] - ISSN 0963-0252 - 27:5(2018), 055008

Full text (Publisher's DOI): https://doi.org/10.1088/1361-6595/AAC0E4

To cite this reference: https://hdl.handle.net/10067/1515460151162165141 
ACCEPTED MANUSCRIPT

\section{Enhancement of plasma generation in catalyst pores with different shapes}

To cite this article before publication: Yu-Ru Zhang et al 2018 Plasma Sources Sci. Technol. in press https://doi.org/10.1088/1361-6595/aac0e4

\section{Manuscript version: Accepted Manuscript}

Accepted Manuscript is "the version of the article accepted for publication including all changes made as a result of the peer review process, and which may also include the addition to the article by IOP Publishing of a header, an article ID, a cover sheet and/or an 'Accepted

Manuscript' watermark, but excluding any other editing, typesetting or other changes made by IOP Publishing and/or its licensors"

This Accepted Manuscript is @ 2018 IOP Publishing Ltd.

During the embargo period (the 12 month period from the publication of the Version of Record of this article), the Accepted Manuscript is fully protected by copyright and cannot be reused or reposted elsewhere.

As the Version of Record of this article is going to be / has been published on a subscription basis, this Accepted Manuscript is available for reuse under a CC BY-NC-ND 3.0 licence after the 12 month embargo period.

After the embargo period, everyone is permitted to use copy and redistribute this article for non-commercial purposes only, provided that they adhere to all the terms of the licence https://creativecommons.org/licences/by-nc-nd/3.0

Although reasonable endeavours have been taken to obtain all necessary permissions from third parties to include their copyrighted content within this article, their full citation and copyright line may not be present in this Accepted Manuscript version. Before using any content from this article, please refer to the Version of Record on IOPscience once published for full citation and copyright details, as permissions will likely be required. All third party content is fully copyright protected, unless specifically stated otherwise in the figure caption in the Version of Record.

View the article online for updates and enhancements. 
Enhancement of plasma generation in catalyst pores with different shapes

\author{
Yu-Ru Zhang ${ }^{\mathrm{a}, \mathrm{b},{ }^{*}}$, Erik C. Neyts ${ }^{\mathrm{b}}$ and Annemie Bogaerts ${ }^{\mathrm{b}}$ \\ ${ }^{a}$ Key Laboratory of Materials Modification by Laser, Ion, and Electron Beams (Ministry of \\ Education), School of Physics, Dalian University of Technology, Dalian 116024, People's \\ Republic of China
}

${ }^{b}$ Research group PLASMANT, Department of Chemistry, University of Antwerp, Universiteitsplein 1, BE-2610 Wilrijk-Antwerp, Belgium

\begin{abstract}
Plasma generation inside catalyst pores is of utmost importance for plasma catalysis, as the existence of plasma species inside the pores affects the active surface area of the catalyst available to the plasma species for catalytic reactions. In this paper, the electric field enhancement, and thus the plasma production inside catalyst pores with different pore shapes is studied with a two-dimensional fluid model. The results indicate that the electric field will be significantly enhanced near tip-like structures. In a conical pore with small opening, the strongest electric field appears at the opening and bottom corners of the pore, giving rise to a prominent ionization rate throughout the pore. For a cylindrical pore, the electric field is only enhanced at the bottom corners of the pore, with lower absolute value, and thus the ionization rate inside the pore is only slightly enhanced. Finally, in a conical pore with large opening, the electric field is characterized by a maximum at the bottom of the pore, yielding a similar behavior for the ionization rate. These results demonstrate that the shape of the pore has a significantly influence on the electric field enhancement, and thus modifies the plasma properties.

* Corresponding author.

E-mail address: yrzhang@dlut.edu.cn
\end{abstract}




\section{Introduction}

Plasma catalysis is gaining increasing interest as an effective method to reduce environmental pollutants and to produce value-added chemicals, more specifically, for hydrocarbon production, methane reforming, $\mathrm{NO}_{\mathrm{x}}$ and $\mathrm{SO}_{\mathrm{x}}$ removal, $\mathrm{CO}_{2}$ conversion and volatile organic compounds (VOCs) abatement [1-4]. Plasma catalysis refers to the combination of nonthermal plasma with a material that has catalytic properties. The main goal of the hybrid plasma catalysis system is to achieve improved process characteristics, i.e., higher energy efficiency and selectivity, compared to the individual processes, i.e., in the plasma or catalysis alone. When this improvement is more than the sum of the individual processes, it is referred to as the synergistic effect of plasma catalysis. Indeed, the presence of the plasma affects the catalyst properties, and vice versa, the dielectric constant and morphology of the catalyst modify the physicochemical plasma properties [5-20]. These modifications are believed to underlay the observed synergistic effect [10].

Dielectric barrier discharges (DBDs) are the most often used type of discharge for plasma catalysis applications. By adding catalysts into a DBD, the electric field is significantly enhanced near the catalyst surface, due to the high local curvature at the pores, or at the contact points between the packing beads [21-24]. Takuma et al. investigated the electric field distribution near and at the contact points for various contact conditions, showing that the electric field is influenced by many factors, i.e., contact angle, relative permittivity and thickness of the dielectric [21]. Chen et al. demonstrated that the void between packing beads is usually not spherical, and the maximum electric field is $10-10^{4}$ times higher than in a spherical void, due to the complicated structure [22]. Fridman demonstrated that in packed bed DBD reactors, the existence of packing pellets may intensify the electric field by a factor of up to 250, depending on their shape, porosity, and dielectric constant [23]. In our previous work, we also observed that the electric field inside catalyst pores is significantly enhanced [24]. Besides, Stollenwerk and Wagner et al. reported that microdischarges have the tendency to start at those positions which are covered by a filament in the previous breakdown, because of the locally enhanced electric field there [25, 26].

In recent years, some theoretical research was performed to investigate the electric field enhancement and the consequent modification on the plasma properties [27-29]. Wang et al. presented computed images of the plasma propagation through a porous dielectric sheet, which is an extreme example of a packed-bed reactor, and they indicated that the electric field enhancement is induced by the porous structure [27]. Using a two-dimensional (2D) fluid 
model, Xiong and Kushner investigated the propagation of an atmospheric pressure ionization wave in flexible capillary tubes, and they concluded that smaller radii of curvature tend to support a larger reduced electric field, resulting in a higher electron temperature and an enhanced ionization rate [28]. Van Laer et al. also employed a 2D axisymmetric fluid model, showing that the plasma in packed bed discharges is initiated at the contact points, because the electric field is strongest there [29].

In addition, also several experimental studies reported on the electric field enhancement due to the surface topography $[30,31,11]$. Takaki et al. focused on the influence of the ferroelectric pellet shape on the $\mathrm{C}_{2} \mathrm{~F}_{6}$ removal. They revealed that by adopting hollow cylindricalshaped pellets, the energy efficiency was 1.5 times higher than in the case of spherical pellets, due to the electric field distortion caused by the sharper edge, which gave rise to lower energy consumption [30]. Yu et al. observed a higher $\mathrm{CO}_{2}$ decomposition efficiency in quartz packed plasmas than in silica gel packed plasmas, due to their different morphologies. Indeed, the sharp edges of the quartz pellets were beneficial for the local electric field enhancement, which was advantageous for the generation of energetic electrons and thus for the high electron impact dissociation rate of $\mathrm{CO}_{2}$ [31] : In order to understand the effect of the support material on $\mathrm{NO}_{\mathrm{x}}$ synthesis, Patil et al. performed experiments with various packing materials, and the higher $\mathrm{NO}_{\mathrm{x}}$ concentration in the case of quartz wool was attributed to its rigid fibrous structure, leading to the enhanced electric field and to high intensity microdischarges [11].

In gas discharges, the electric field plays a pivotal role in determining the plasma composition, as it modifies the electron energy distribution function, and this in turn affects the electron impact reaction rates. Indeed, an enhanced electric field results in a higher electron temperature, which is more favorable for electron impact collisions, and the latter are responsible for the production of various reactive species. Therefore, it is of significant importance to understand the mechanisms of the electric field enhancement due to the catalyst morphology or the pore shape, and the consequent influence on the plasma characteristics. In this work, we employ a 2D fluid model to investigate the plasma generation near and inside catalyst pores for various pore shapes. In particular, we systematically study the influence of the pore shape on the electric field distribution, as well as on the electron impact ionization rate, which is a measure for the plasma generation. Indeed, it is important to have a good insight in the electric field enhancement, as it affects the plasma composition, and thus the efficiency of the plasma catalytic processes. 


\section{Description of the model}

In this work, we employed the Comsol Multiphysics software to perform 2D simulations of a DBD with various pore shapes. Comsol is implemented on an unstructured mesh, to enable the simultaneous resolution of processes occurring at different spatial scales [32].

The model used in this investigation is the same as described in [24]. Briefly, the conservation equations solved for multi-fluid charged and neutral species are

$$
\begin{gathered}
\frac{\partial n_{e}}{\partial t}=-\nabla \cdot \boldsymbol{\Gamma}_{\mathbf{e}}+R_{e} \\
\rho \frac{\partial}{\partial t}\left(w_{k}\right)=-\nabla \cdot\left(\rho w_{k} \mathbf{V}_{\mathbf{k}}\right)+R_{k} .
\end{gathered}
$$

Here, $n_{e}, \Gamma_{\mathrm{e}}$ and $R_{e}$ are the electron number density, electron flux and electron source term, respectively. $\rho$ refers to the mass density of the gas mixture. $\mathcal{W}_{k}, \mathbf{V}_{\mathrm{k}}$ and $R_{k}$ are the mass fraction, velocity and source term of the heavy species $(\mathrm{k})$. Note that the drift-diffusion approximation is applied for electrons and ions, while for neutral species, only the diffusion term is included.

The electron energy equation is solved to obtain the electron energy density $n_{\varepsilon}$

$$
\frac{\partial n_{\varepsilon}}{\partial t}=-\mathbf{E} \cdot \boldsymbol{\Gamma}_{\mathrm{e}}-\nabla \cdot \boldsymbol{\Gamma}_{\varepsilon}+R_{\varepsilon},
$$

where $\Gamma_{\varepsilon}$ is the electron energy flux, and $R_{\varepsilon}$ is, the energy source term, which includes the energy exchange due to electron impact collisions (i.e., excitation, ionization, etc.). In a DBD, the gas might locally heat up under certain conditions, i.e., in the vicinity of structures with high curvature. However, since most of the input energy goes to heating of the electrons, the gas temperature will not increase significantly, also due to cooling by the gas flow [29]. Therefore, we assume a constant gas temperature of $300 \mathrm{~K}$ in the model, and no energy balance equation is needed for the ions and neutral species.

The time-dependent electric potential and electric field distributions are determined by solving the Poisson equation throughout the entire computational domain.

$$
\nabla^{2} V=-\frac{\rho_{\text {charge }}}{\varepsilon_{0} \varepsilon_{r}}
$$

where $\rho_{\text {charge }}$ is the space charge density, which is zero inside dielectric materials, $\varepsilon_{0}$ is the vacuum permittivity, and $\varepsilon_{r}$ is the relative permittivity only used for the dielectric region. 
Although molecular gases, such as air mixtures or $\mathrm{CO}_{2} / \mathrm{CH}_{4}$, are typically used in environmental applications of plasma catalysis, the calculations are performed in a helium DBD in this work, to keep the chemistry simple. This reduces the computational cost, and we can focus on the electric field enhancement. The species considered in the model include He, $\mathrm{He}^{\mathrm{M}}, \mathrm{He}_{2}{ }^{\mathrm{M}}, \mathrm{He}^{+}, \mathrm{He}_{2}{ }^{+}$and electrons. The electrons collide with $\mathrm{He}$ atoms, generating $\mathrm{He}^{\mathrm{M}}$ and $\mathrm{He}^{+}$upon excitation and ionization. $\mathrm{He}^{\mathrm{M}}$ denotes a combined metastable level, which consists of the $\mathrm{He}\left(2^{1} \mathrm{~S}\right)$ and $\mathrm{He}\left(2^{3} \mathrm{~S}\right)$ levels, and $\mathrm{He}_{2}{ }^{\mathrm{M}}$ represents the molecular metastable $\mathrm{He}_{2}\left(\mathrm{a}^{3} \mathrm{Su}\right)$. By colliding with electrons, the $\mathrm{He}^{\mathrm{M}}$ species either quench to the ground state or produce electrons during Penning ionization. The recombination loss mechanisms of ions are also taken into account in the model. The rate coefficients for the electron impact reactions mentioned above are calculated by BOLSIG+, based on the corresponding collision cross sections [33]. The full chemistry set is listed in Table 1, together with the rate coefficients for the heavy particle reactions and recombination reactions.

In addition, a simple surface mechanism is considered, in which the $\mathrm{He}^{+}$and $\mathrm{He}_{2}{ }^{+}$ions are neutralized to ground state helium atoms, with a probability of 1 . The sticking coefficients for $\mathrm{He}^{\mathrm{M}}$ and $\mathrm{He}_{2}{ }^{\mathrm{M}}$ are set as 1 at the walls. Secondary electron emission induced by ion impact is also considered, with a probability of 0.05 [29]. Sputtering is not included in the present model.

Table 1. The reactions for the He plasma included in the model. The electron temperature $T_{e}$ and the gas temperature $\mathrm{T}_{\mathrm{g}}$ are in $\mathrm{eV}$.

\begin{tabular}{clll}
\hline No. & \multicolumn{1}{c}{ Reaction } & Rate Coefficient & Reference \\
\hline $\mathrm{R} 1$ & $\mathrm{e}+\mathrm{He} \rightarrow \mathrm{He}+\mathrm{e}$ & From cross-section & 34 \\
$\mathrm{R} 2$ & $\mathrm{e}+\mathrm{He} \rightarrow \mathrm{He}^{+}+2 \mathrm{e}$ & From cross-section & 34 \\
$\mathrm{R} 3$ & $\mathrm{e}+\mathrm{He} \rightarrow \mathrm{He}^{\mathrm{M}}+\mathrm{e}$ & From cross-section & 34 \\
$\mathrm{R} 4$ & $\mathrm{e}+\mathrm{He}^{\mathrm{M}} \rightarrow \mathrm{He}+\mathrm{e}$ & From cross-section & 34 \\
$\mathrm{R} 5$ & $\mathrm{e}+\mathrm{He}^{\mathrm{M}} \rightarrow \mathrm{He}^{+}+2 \mathrm{e}$ & From cross-section & 34 \\
$\mathrm{R} 6$ & $\mathrm{e}+\mathrm{He}_{2}{ }^{\mathrm{M}} \rightarrow 2 \mathrm{He}+\mathrm{e}$ & $3.8 \times 10^{-9} \mathrm{~cm}^{3} \mathrm{~s}^{-1}$ & 35 \\
$\mathrm{R} 7$ & $\mathrm{e}+\mathrm{He}_{2}{ }^{\mathrm{M}} \rightarrow \mathrm{He}_{2}{ }^{+}+2 \mathrm{e}$ & $9.75 \times 10^{-10} \mathrm{~T}_{\mathrm{e}}{ }^{0.71} \mathrm{e}^{-3.4 / \mathrm{Te}} \mathrm{cm}^{3} \mathrm{~s}^{-1}$ & 36 \\
$\mathrm{R} 8$ & $2 \mathrm{e}+\mathrm{He}^{+} \rightarrow \mathrm{He}^{\mathrm{M}}+\mathrm{e}$ & $1.5 \times 10^{-20}\left(\mathrm{~T}_{\mathrm{e}} / \mathrm{T}_{\mathrm{g}}\right)^{-4} \mathrm{~cm}^{6} \mathrm{~s}^{-1}$ & 35,37
\end{tabular}


R9

$$
\mathrm{e}+\mathrm{He}_{2}{ }^{+} \rightarrow \mathrm{He}^{\mathrm{M}}+\mathrm{He}
$$

$5.0 \times 10^{-9}\left(\mathrm{~T}_{\mathrm{e}} / \mathrm{T}_{\mathrm{g}}\right)^{-1} \mathrm{~cm}^{3} \mathrm{~s}^{-1}$

$\mathrm{R} 10 \quad 2 \mathrm{e}+\mathrm{He}_{2}{ }^{+} \rightarrow \mathrm{He}^{*}+\mathrm{He}+\mathrm{e}$

$$
\rightarrow \mathrm{He}_{2}{ }^{*}+\mathrm{e}
$$

$4.0 \times 10^{-20}\left(\mathrm{~T}_{\mathrm{e}} / \mathrm{T}_{\mathrm{g}}\right)^{-4} \mathrm{~cm}^{6} \mathrm{~s}^{-1}$

$\mathrm{R} 11 \mathrm{e}+\mathrm{He}_{2}{ }^{+}+\mathrm{He} \rightarrow \mathrm{He}^{*}+2 \mathrm{He}$

$$
\rightarrow \mathrm{He}_{2}{ }^{*}+\mathrm{He}
$$

$$
5.0 \times 10^{-27}\left(\mathrm{~T}_{\mathrm{e}} / \mathrm{T}_{\mathrm{g}}\right)^{-1} \mathrm{~cm}^{6} \mathrm{~s}^{-1}
$$

$\mathrm{R} 12 \mathrm{He}^{+}+2 \mathrm{He} \rightarrow \mathrm{He}_{2}{ }^{+}+\mathrm{He}$

$1.4 \times 10^{-31}\left(\mathrm{~T}_{\mathrm{g}} / 0.025\right)^{-0.6} \mathrm{~cm}^{6} \mathrm{~s}^{-1}$

$$
\mathrm{R} 13 \quad \mathrm{He}^{\mathrm{M}}+2 \mathrm{He} \rightarrow \mathrm{He}_{2}{ }^{\mathrm{M}}+\mathrm{He}
$$

$2.5 \times 10^{-34} \mathrm{~cm}^{6} \mathrm{~s}^{-1}$

$\mathrm{R} 14 \quad \mathrm{He}^{\mathrm{M}}+\mathrm{He}^{\mathrm{M}} \rightarrow \mathrm{He}^{+}+\mathrm{He}+\mathrm{e}$

$4.5 \times 10^{-10} \mathrm{~cm}^{3} \mathrm{~s}^{-1}$

$$
\rightarrow \mathrm{He}_{2}{ }^{+}+\mathrm{e}
$$

$1.05 \times 10^{-9} \mathrm{~cm}^{3} / \mathrm{s}^{-1}$

R15

$$
\mathrm{He}_{2}{ }^{\mathrm{M}}+\mathrm{He}^{\mathrm{M}} \rightarrow \mathrm{He}^{+}+2 \mathrm{He}+\mathrm{e}
$$

$5.0 \times 10^{-10} \mathrm{~cm}^{3} \mathrm{~s}^{-1}$

$$
\rightarrow \mathrm{He}_{2}{ }^{+}+\mathrm{He}+\mathrm{e}
$$

$2.0 \times 10^{-9} \mathrm{~cm}^{3} \mathrm{~s}^{-1}$

$$
\mathrm{R} 16 \quad \mathrm{He}_{2}{ }^{\mathrm{M}}+\mathrm{He}_{2}{ }^{\mathrm{M}} \rightarrow \mathrm{He}^{+}+3 \mathrm{He}+\mathrm{e} \quad 3.0 \times 10^{-10} \mathrm{~cm}^{3} \mathrm{~s}^{-1}
$$

$$
\rightarrow \mathrm{He}_{2}^{+}+2 \mathrm{He}+\mathrm{e} \quad 1.2 \times 10^{-9} \mathrm{~cm}^{3} \mathrm{~s}^{-1}
$$

\section{Results and discussion}

In this paper, we study in detail the effect of the pore shape on the electric field enhancement, and accordingly its influence on the plasma generation. The investigations are performed for a DBD configuration. The discharge region is $0.2 \mathrm{~mm}$ in width, and $2 \mathrm{~mm}$ in height. The top electrode is powered by an alternating current source $V=V_{0} \sin (2 \pi f t)$, at a frequency $f$ of 25 $\mathrm{kHz}$, and a voltage $V_{0}$ of $20 \mathrm{kV}$, and the bottom electrode is grounded. The discharge gap is bounded by two dielectric layers, with thickness of $1 \mathrm{~mm}$ on the top and $0.5 \mathrm{~mm}$ at the bottom. In order to study the influence of the pore shape on the plasma characteristics, the bottom dielectric plate, i.e., $\mathrm{Al}_{2} \mathrm{O}_{3}\left(\varepsilon_{\mathrm{r}}=9\right)$, is porous. Three different pore shapes are considered, i.e., a conical pore with small opening, a cylindrical pore and a conical pore with large opening (see figure 1). These three pore shapes are not necessarily the same as in actual catalyst materials, but we think they represent some extreme conditions, which can help us to understand in general the influence of the pore shape on the plasma generation. Note that the edge and bottom of the pores are rounded to reduce the computational cost, with the curvature 
fixed at $300 \mathrm{~mm}^{-1}$. For the conical pore with small opening (see figure 1(a)), the width of the pore is $5 \mu \mathrm{m}$ on the top and $50 \mu \mathrm{m}$ at the bottom. For the cylindrical pore shown in figure 1(b), the width of the pore is fixed at $50 \mu \mathrm{m}$. In figure 1(c), the top width of the conical pore is $50 \mu \mathrm{m}$, while the bottom has a width of $5 \mu \mathrm{m}$. Besides, the depth of all these pores is fixed at $100 \mu \mathrm{m}$. Although the geometry is symmetric along the center of the pores in this work, we wanted to build a model that is more generally valid, in order to be able to also investigate asymmetric geometries in the future. Therefore, the model is built in Cartesian coordinates, and we simulate the entire geometry.

\subsection{Electron impact ionization rate - a measure for the plasma generation}

Figure 1 shows the calculated time-averaged ionization rates near and inside the pore for the various pore shapes. For the conical pore with small opening (figure 1(a)), the maximum ionization rate appears at the opening of the pore, but the ionization rate at the bottom of the pore is also high. For the cylindrical pore (figure 1(b)), the maximum ionization rate appears at the corners of the pore bottom, and it is also quite high at the center of the pore, although about a factor of 4 lower than the maximum in figure 1(a). Also above the pore, there is still some local enhancement (i.e., $1.5 \times 10^{25} \mathrm{~m}^{-3} \mathrm{~s}^{-1}$ ), which is not visible in figures 1(a) and 1(c). For the conical pore with large opening (figure 1(c)), the maximum ionization rate only appears at the bottom of the pore, and the maximum value is also somewhat higher than in figure 1(a).

By integrating the ionization rate inside the pore, we obtained a value of $8.68 \times 10^{11} \mathrm{~s}^{-1}$ for a conical pore with small opening, $6.93 \times 10^{11} \mathrm{~s}^{-1}$ for a cylindrical pore, and $4.19 \times 10^{11} \mathrm{~s}^{-1}$ for a conical pore with large opening. Since a higher ionization rate most probably also corresponds to a larger generation of reactive plasma species inside the pores, which will be available for surface reactions at the catalyst surface, we expect the conical pore with small opening to perform better under the conditions investigated in this work.

In order to illustrate the time-dependence of the ionization rate and to gain more insight into the plasma generation for various pore shapes, the electron impact ionization rate along the centerline of the pore at four different times within one cycle of the applied voltage is shown in figure 2 (see the inset in figure 2(a) for the sinusoidal voltage profile). Note that $y=$ $-0.1 \mathrm{~mm}$ corresponds to the bottom of the pore. For a conical pore with small opening (Fig. 2(a)), the ionization rate is by far the highest at $\varphi=0$ (see black solid curve), and it appears at the opening of the pore. This profile is responsible for the peak value of the time-averaged 
ionization rate in figure 1(a). At the other time phases, the ionization rate is much lower, and exhibits different shapes. For instance, at $\varphi=0.5 \pi$, the ionization rate shows a similar profile as the one at $\varphi=0$. Although the absolute value is about two orders of magnitude lower than at $\varphi=0$, it still contributes to some extent to the maximum ionization rate in figure 1(a). As the time phase increases to $\varphi=\pi$, the maximum shifts from the opening of the pore to the bottom. Subsequently, the ionization rate inside the pore increases significantly, but the value at the opening of the pore is still higher than at the bottom. A similar behavior is observed at $\varphi=1.5 \pi$, but the absolute value is again much lower (i.e., it is only about $1.7 \times 10^{25} \mathrm{~m}^{-3} \mathrm{~s}^{-1}$ at $\varphi=1.5 \pi$, while it is around $5.4 \times 10^{26} \mathrm{~m}^{-3} \mathrm{~s}^{-1}$ at $\left.\varphi=1.1 \pi\right)$. The non-negligible time-averaged ionization rate inside and at the bottom of the pore in figure 1 (a) is thus attributed to the ionization rate distributions during this second half period of the applied voltage.

For a cylindrical pore (Fig. 2(b)), the ionization rates at $\varphi=0$ and $\varphi=\pi$ are comparable in magnitude, but the maximum is reached at different positions, i.e., it is at the pore opening and inside the sheath at $\varphi=0$, while it is inside the pore and more towards the bottom at $\varphi=\pi$. At $\varphi=0.5 \pi$ and $\varphi=1.5 \pi$, the ionization rate profiles are similar to those at $\varphi=0$ and $\varphi=\pi$, respectively, but the values are a factor of 30-100 lower. These distributions are responsible for the time-averaged ionization rate, which does not show a pronounced maximum, but rather a broad distribution both inside and above the pore in Fig. 1(b). Note that the maximum at $\varphi=\pi$ is slightly higher than that at $\varphi=0$, but both of them are a factor of 2-2.5 lower than at $\varphi=0$ in a conical pore with small opening. Therefore, the time-averaged ionization rate inside the cylindrical pore (see figure $1(b)$ ) is much lower than the peak value at the pore opening in figure 1(a). In addition, the time-averaged ionization rate is slightly enhanced at the corners of the cylindrical pore bottom, due to the strong electric field there, as will be explained below.

For a conical pore with large opening (Fig. 2(c)), the ionization mainly takes place at the pore opening and inside the sheath at $\varphi=0$, with a broad maximum of about $4.1 \times 10^{25} \mathrm{~m}^{-3} \mathrm{~s}^{-1}$, but it decreases significantly as a function of time and becomes negligible at $\varphi=0.5 \pi$, with a maximum of only about $5.2 \times 10^{23} \mathrm{~m}^{-3} \mathrm{~s}^{-1}$. However, a pronounced peak is observed at the bottom of the pore at $\varphi=\pi$, with values much higher than those at the other time phases, i.e., around $5 \times 10^{26} \mathrm{~m}^{-3} \mathrm{~s}^{-1}$. This gives rise to the time-averaged ionization rate distribution in Fig. 1(c), with a pronounced maximum at the bottom of the pore. Besides, the higher absolute 
value of the ionization rate at $\varphi=\pi$ in figure 2(c) also leads to the more substantial timeaveraged ionization rate in figure 1(c) compared to those in figures 1(a) and 1(b).

\subsection{Elucidating the plasma behavior - Electric field and plasma density distributions}

The evolution of the ionization rate with time can be understood by examining the electric field distributions combined with the electron density distributions. The electric field distributions for the three different pore shapes are plotted in figures 3,5 and 7 , while the corresponding electron density distributions are presented in figures 4, 6 and 8. Note that in figures 3, 5 and 7, we plot the absolute values of the total electric field, which is calculated as $\sqrt{E_{x}{ }^{2}+E_{y}{ }^{2}}$. The vectors indicate the direction of the electric field, based on the $\mathrm{x}$ and $\mathrm{y}$ components $\left(\mathrm{E}_{\mathrm{x}}\right.$ and $\left.\mathrm{E}_{\mathrm{y}}\right)$. These vectors are plotted with constant length, as they only indicate the direction, while the electric field magnitude is indicated by the color scale. Likewise, in figures 4, 6 and 8, besides the electron density profiles, illustrated by the color scale, both the direction and the absolute value of the electron flux can be deduced from the vectors, whose lengths are proportional to the flux magnitudes.

\section{(a) Conical pore with small opening}

For a conical pore with small opening (figure 3), the potential at the top dielectric layer is positive at $\varphi=0$, whereas it is negative at the bottom dielectric layer due to the charge accumulation. Therefore, the electric field induced by this potential difference is directed downwards, as can be seen from the vectors in figure 3(a). Besides, due to the small size of the pore opening, the electric field is very strong there. It is indeed well known that the electric field is enhanced near tip-like structures, and the enhancement can be up to 1000 or more for high aspect ratio structures [40]. The electrons are heated by the strong electric field at the opening of the pore and they ionize the gas significantly, leading to the high ionization rate at the pore opening at $\varphi=0$ (cf. figure 2(a)). Note that for the same curvature, the ionization rate at the opening of the conical pore would be significantly reduced if the size of the opening increases. This is because for smaller opening, the electric potential decreases rapidly oyer a shorter distance, leading to a stronger electric field.

At $\varphi=0.5 \pi$ (figure 3(b)), the applied voltage at the top electrode is $20 \mathrm{kV}$ (see inset in figure 2(a)). Although most of the potential drops in the dielectric, the larger potential difference gives rise to the stronger axial electric field $E_{y}$. Besides, the lateral electric field $E_{x}$ also becomes stronger, resulting in a higher total electric field than at $\varphi=0$ (cf. figures 3(b) 
and 3(a)). Note that although the electrons thus gain more energy at the pore opening at $\varphi=$ $0.5 \pi$, the maximum ionization rate is about two orders of magnitude lower than at $\varphi=0$, due to the significantly lower electron density, as will be discussed in detail below.

At $\varphi=\pi$, although the applied voltage at the top electrode is $0 \mathrm{~V}$, the positive charge accumulates at the bottom of the pore, leading to an upward electric field, as indicated by the vectors in figure 3(c), and it is the strongest at the bottom corners of the pore. The electric field distribution explains why the ionization rate is higher at the bottom of the pore than at the opening at $\varphi=\pi$, because the stronger electric field gives more electron heating, and thus more electrons can contribute to the ionization.

At $\varphi=1.5 \pi$ (figure $3(\mathrm{~d})$ ), the electric field is again characterized by a maximum at the bottom corners of the pore, but the maximum value is somewhat lower. Moreover, the electric field in the sheath near the bottom dielectric plate is very weak, and it is directed downwards due to the negative charge accumulation. Note that although the electric field at the pore opening is slightly lower than at the bottom corners (i.e., $3.6 \times 10^{6} \mathrm{~V} / \mathrm{m}$ vs. $6.5 \times 10^{6} \mathrm{~V} / \mathrm{m}$ ), the maximum ionization rate takes place at the pore opening, due to the higher electron density there, as will be shown below.

The electron density distributions, together with the electron flux indicated by vectors, near and inside the conical pore with small opening are shown in figure 4. Note that different color scales are used to clearly see the profiles. By comparing the electron density at these four different times, it is clear that although all of the maxima appear in the sheath above the pore, the distributions are strikingly different, both in shape and in absolute value. At $\varphi=0$, the plasma generation, visualized by the electron impact ionization rate (figure 2(a)), mainly takes place at the pore opening, due to the high electron temperature caused by the strong electric field. However, the electrons are accelerated out of the pore due to the downward electric field (cf. figure 3(a)), especially at the pore opening, which is clear from the longer vectors in figure 4(a), leading to a higher electron density above the pore.

From figure 4(b), it is elear that the maximum electron density at $\varphi=0.5 \pi$ is about two orders of magnitude lower than at $\varphi=0$, which can be attributed to the strong electric field near the bottom dielectric plate. Indeed, the electrons are accelerated towards the bulk plasma, leaving a region with low electron density here. Although the electron heating is more effective due to the stronger electric field, the plasma generation process is limited because fewer electrons are available to ionize the gas. Therefore, the ionization rate is characterized 
by a peak at the pore opening, but with a much lower value than at $\varphi=0$, as shown in figure 2(a) above.

At $\varphi=\pi$, the ionization rate exhibits a pronounced peak in the sheath near the top dielectric plate due to the strong electric field (not shown here). As a consequence, the electrons are accelerated away from the top dielectric plate, and the maximum electron density appears in the bulk region. Since the electric field near the bottom dielectric is directed upwards (see figure 3(c)), the electrons flow downwards due to migration, as is obvious from figure 4(c). Moreover, the low electron density inside the pore is responsible for the weak plasma production process. However, the ionization rate inside the pore, especially at the bottom of the pore, is still higher than above the pore, which can again be explained by the higher electron temperature resulting from the enhanced electric field.

Compared to the result shown at $\varphi=\pi$, the electron density in the sheath above the bottom dielectric at $\varphi=1.5 \pi$ is much lower, due to the downward electric field, as illustrated in figure 3(d) above, accelerating the electrons towards the bulk. Because of the upward electric field in the sheath near the top dielectric layer and in the bulk region (not shown here), the electrons are also accelerated downwards from the top dielectric, and this results in a pronounced peak in the electron density above the pore, as is obvious from figure 4(d). The electrons thus flow into the pore through the pore opening, under the influence of diffusion and migration, which can be clearly deduced from the direction of the vectors in figure 4(d). As a consequence, the electron density inside the pore is quite high (not so clearly visible in figure 4(d), but note the different color scale in figure 4(d) compared to the other figures), together with the rather strong electric field (see figure 3(d)), and this results in a considerable ionization rate throughout the pore.

\section{(b) Cylindrical pore}

For a cylindrical pore, the electric field exhibits quite different distributions from the conical pore with small opening, as shown in figure 5. At $\varphi=0$ (see figure 5(a)), the electric field at the pore opening is not as strong as that observed in figure 3(a), because the larger pore opening limits the electric field enhancement. In addition, the electric field throughout the pore is quite strong, which efficiently heats the electrons. Since the electric field inside the pore is directed downwards, the energetic electrons are accelerated upwards, which is clear from the higher electron flux indicated by the longer vectors in figure 6(a). Therefore, the 
ionization rate is characterized by a dominant peak at the opening of the pore (see figure 2(b) above).

The electric field at $\varphi=0.5 \pi$ reaches a similar maximum value at the corners of the pore opening, but the value inside the pore is slightly lower than at $\varphi=0$ (see figure 5(b)). Again, the electrons near the bottom dielectric are significantly influenced by this strong downward electric field, accelerating the electrons upward, leading to a much lower electron density inside and above the pore, and thus the ionization rate is negligible (see figure 2(b)).

At $\varphi=\pi$, the electric field at the bottom corners of the pore is very strong and points upwards, thus attracting the electrons towards the bottom (see figures 5(c) and 6(c)). These electrons are heated inside the pore, especially at the bottom corners, which leads to a pronounced ionization rate there, i.e., slightly higher than at $\varphi=0$ (see figure 2(b)). At $\varphi=$ $1.5 \pi$, although the maximum value of the electric field at the bottom corners is slightly higher than at $\varphi=\pi$, the electric field at the center of the pore is somewhat weaker. This weak electric field and the corresponding low electron density are responsible for the very low ionization rate profile, which is about more than one order of magnitude lower than at $\varphi=\pi$.

\section{(c) Conical pore with large opening}

Figure 7 shows the electric field distributions at four different times within one cycle for a conical pore with large opening. At $\varphi=0$ (see figure 7(a)), the electric field increases monotonously from the top of the pore to the bottom. However, as the electric field at the bottom of the pore is only a factor of 2-3 higher than at the opening, whereas the electron density is about two orders of magnitude lower (see figure 8(a)), the ionization rate exhibits a maximum at the pore opening and inside the sheath, as shown in figure 2(c) above. At $\varphi=$ $0.5 \pi$, although the electric field exhibits a similar behavior (see figure 7(b)), the electron density is two orders of magnitude lower than at $\varphi=0$ (cf. figure $8(\mathrm{~b})$ ), explaining the negligible ionization rate (cf. figure 2(c)). Note that the electric field distribution is strikingly different from those in a conical pore with small opening and in a cylindrical pore, indicating the significant influence of the pore shape on the electric field distribution. For instance, for a conical pore with small opening (see figure 3(a)), the electric field reaches its maximum at the pore opening, and it decreases monotonously towards the bottom of the pore, while for a cylindrical pore (see figure 5(a)), the electric field enhancement is more limited due to the larger pore opening, and in a conical pore with large opening, the electric field is most 
pronounced in the narrow bottom. Indeed, the electric field is typically stronger at a tip-like structure.

At $\varphi=\pi$, the electric field in the top half of the pore is lower than at $\varphi=0$ and $\varphi=0.5 \pi$, but it increases rapidly near the bottom of the pore, where the absolute value is about three times higher. This is again different from the behavior observed in a conical pore with small opening. Indeed, although in figure 3(c), the electric field also reaches its maximum at the bottom corners of the pore at $\varphi=\pi$, the value in the center of the pore is much higher here than in figure 7(c). In case of the conical pore with large opening, abundant electrons are attracted by this strong electric field towards the bottom and heated there, thus causing pronounced ionization at the bottom, as is clear from figure 2(c) above. At $\varphi=1.5 \pi$ (cf. figure 7(d)), the electric field at the bottom is even stronger, but the value in the top half of the pore is somewhat lower than at $\varphi=\pi$. Besides, the electron density inside the pore is somewhat lower in figure $8(\mathrm{~d})$, so the ionization rate distribution inside the pore at $\varphi=1.5 \pi$ is about one order of magnitude lower.

\section{(d) Time-averaged profiles: comparison between the different pore shapes}

The time-averaged electric field distributions for the three different pore shapes are compared in figure 9. This comparison allows us to obtain more insight in the influence of the pore shape on the electric field enhancement. For a conical pore with small opening, the electric field is the strongest both at the opening and bottom corners of the pore, as shown in figure $9(\mathrm{a})$, and the power consumption per unit depth is about $2.12 \times 10^{-1} \mathrm{~W} / \mathrm{m}$. In the sheath above the pore, the electric field is directed downwards, as expected, and it reaches its maximum value at the pore opening. Inside the pore, the absolute value of the y component of the electric field decreases first and then it increases, with a minimum at about $\mathrm{y}=-0.07 \mathrm{~mm}$, and thus the total electric field is here aligned along the $\mathrm{x}$ axis. At the bottom of the pore, the electric field is directed upwards, which can be attributed to the higher potential resulting from the positive charge accumulation there.

For a cylindrical pore, as shown in figure 9(b), the time-averaged electric field is much weaker, which is only enhanced at the bottom corners of the pore, and the power consumption per unit depth is about $1.53 \times 10^{-1} \mathrm{~W} / \mathrm{m}$. In addition, the electric field is always directed downwards as expected, even at the bottom of pore, indicating that the effect of the pore shape on the electrical structure of the discharge is limited due to the large pore opening. 
From figure 9(c), it is clear that the absolute value of the electric field inside a conical pore with large opening increases monotonously from the opening till the bottom. The maximum indeed appears at the bottom of the pore, and its value is even somewhat higher than at the pore opening in figure 9(a). Although the electric field at the center of the pore is stronger than in a cylindrical pore, the enhancement is not as obvious as in a conical pore with small opening, and the power consumption per unit depth is about $8.36 \times 10^{-2} \mathrm{~W} / \mathrm{m}$ in this case. The different influences of the pore shape on the electric field distribution affect the transient behavior of electrodynamics, and thus modify the plasma generation, as explained in previous sections.

The electron temperature distributions are largely determined by the electric field, as the electrons can gain more energy from the stronger electric field, and this gives rise to the higher electron temperature. Therefore, for a conical pore with small opening, the maximum electron temperature appears both at the opening and bottom corners of the pore (figure 10(a)). Besides, the electron temperature is around $10 \mathrm{eV}$ throughout the pore, leading to the higher ionization rate (see figure 1(a) above). For a cylindrical pore, the weaker electric field is responsible for the lower electron temperature (figure 10(b)), and so is the ionization rate (figure 1(b) above). In figure 10(c), the electron temperature reaches its maximum at the bottom of the pore, and the value is even higher than the maximum in figure 10(a), due to the strong electric field at the bottom. However, at the pore opening, the electron temperature is only about $5 \mathrm{eV}$, thus the ionization rate is more than one order of magnitude lower than at the bottom. Because electron impact ionization and Penning ionization contribute significantly to the ion formation, the total ion density profile (not shown here) also depends on the electron temperature distribution. For a conical pore with small opening, the total ion density is high both inside the pore and at the pore opening, whereas for the other two cases, the maximum is observed at the center of the pore.

Figure 11 illustrates the time-averaged electron density profiles for the three different pore shapes. It is clear that the maximum electron density appears above the pore in all cases, and the absolute value for a conical pore with small opening is somewhat lower than in the other two cases. Besides, the electron density inside the pore is very low. Indeed, although the plasma generation is enhanced inside the pore (see figure 1 above), the electrons are accelerated out of the pore due to the downward electric field (see figure 9). Especially in figure 11 (a), the electron density is even lower than in the other two cases. However, the ionization rate is obviously enhanced in figure 1(a), due to the strong electric field at the small pore opening, as shown in figure 9(a). Vice versa, although the electron density inside a 
cylindrical pore is slightly higher, the ionization rate enhancement is limited because of the weaker electric field. The difference between the spatial profiles of ionization rate and electron density is because although the ionization mainly takes place inside the pore, after these electrons are generated, they move due to diffusion and migration. Therefore, the higher electron density appears at the bulk region (not shown here), and this leads to a difference between the distributions of the electron density and the ionization rate near the bottom dielectric.

Indeed, a fluid model might be at the limit of accuracy, especially when the characteristic dimension is too small, or the particle density is too low. However, in this paper, the fluid model is applied for the whole discharge region of $2 \mathrm{~mm}$ in height. In the bulk region, the electron density is in the order of $10^{17} \mathrm{~m}^{-3}$, and the mesh size is about $15 \mu \mathrm{m}$, which is much larger than the electron mean free path (i.e., about $0.5 \mu \mathrm{m}$ ). Only for the conical pore with small opening, the mesh is locally refined at the pore opening, with a resolution of about $1 \mu \mathrm{m}$. Therefore, we believe there are in general enough collisions in the whole discharge region for the electrons to reach thermal equilibrium, and the fluid model should be valid, as was also demonstrated by Kushner and coworkers, for pores of several $\mu \mathrm{m}$, with similar electron density near the pore as in our case [41-43].

The distributions of the $\mathrm{He}^{\mathrm{M}}$ density and $\mathrm{He}_{2}{ }^{\mathrm{M}}$ density obtained for the different pore shapes are shown in figures 12 and 13, respectively. In this work, the simulation runs till $10^{-3}$ $\mathrm{s}$, which is longer than the decay time of metastables (i.e., about $6.7 \times 10^{-4} \mathrm{~s}$ ), to make sure that they reach steady state values. Note that the influence of the pore shape on the $\mathrm{He}^{\mathrm{M}}$ density profile is more obvious than that on the $\mathrm{He}_{2}{ }^{\mathrm{M}}$ density. This is because electron impact excitation is an important generation mechanism of $\mathrm{He}^{\mathrm{M}}$ species, so the $\mathrm{He}^{\mathrm{M}}$ density profile is more sensitive to the electron temperature distribution. From figure 12, it is clear that the $\mathrm{He}^{\mathrm{M}}$ density is very high throughout the conical pore with small opening, whereas for the conical pore with large opening, the maximum only appears at the bottom of the pore. For a cylindrical pore, the $\mathrm{He}^{\mathrm{M}}$ density reaches its maximum at the center of the pore, but the value is much lower than in the other two cases. As the $\mathrm{He}_{2}{ }^{\mathrm{M}}$ species are formed through the recombination and collisions between $\mathrm{He}^{\mathrm{M}}$ and $\mathrm{He}$ atoms, the maximum $\mathrm{He}_{2}{ }^{\mathrm{M}}$ density appears above the pore in all cases, as shown in figure 13.

In addition, near the bottom dielectric, the $\mathrm{He}^{\mathrm{M}}$ density and $\mathrm{He}_{2}{ }^{\mathrm{M}}$ density are much higher than the electron density, which is different from experimental results from literature [39, 44]. This is because the electron temperature is much higher near the two dielectric layers than in 
the bulk region due to the strong electric field in the sheath, and this gives rise to the higher electron impact excitation rate, and consequently the higher $\mathrm{He}^{\mathrm{M}}$ density and $\mathrm{He}_{2}{ }^{\mathrm{M}}$ density. Although the ionization also mainly takes place near the dielectrics, the electrons move towards the bulk region due to migration, and this leads to the lower electron density near the dielectrics, as shown in figure 11. However, the opposite trend is observed in the bulk region, with a higher electron density than $\mathrm{He}^{\mathrm{M}}$ density and $\mathrm{He}_{2}{ }^{\mathrm{M}}$ density (see figure 14, the negative $\mathrm{y}$-values indicate the bottom of the pore and $\mathrm{y}=2 \mathrm{~mm}$ indicates the top dielectric plate).

Finally, the surface charge density at the bottom dielectric and at the pore walls is presented in figure 15. It is clear that a positive charge is accumulated on the dielectric surface outside the pore in all cases, due to the electric field there, which accelerates the positive ions downwards (see figure 9). The charge density reaches its maximum at the edge of the pore opening, and then it drops to negative values. Inside the pore, the surface charge density behaves differently for the various pore shapes. At the sidewalls of the conical pore with small opening (black solid line), the charge density increases at the pore bottom, and two peaks are observed at normalized positions of 0.45 and 0.55 . This is because the positive ions are accelerated by the strong electric field towards the sidewalls and the bottom corners of the pore (see figure 9(a)). The dominant influence of migration due to the electric field on the surface charge density can also be illustrated by the pronounced peak at the bottom of the conical pore with large opening (blue dotted line), where the charge density is about $1.2 \times 10^{-3}$ $\mathrm{C} / \mathrm{m}^{2}$. For a cylindrical pore (red dashed line), the charge density at the sidewalls decreases when moving from the top of the pore to the bottom, indicating that the thermal motion of the electrons has a more important effect on the surface charge density. Besides, the charge density becomes slightly higher again at the bottom corner, because the electric field is enhanced there.

\section{Conclusion}

In this paper, we studied the influence of the pore shape on the electric field enhancement, and thus on the transient behavior of electrodynamics and plasma generation, which is of great interest for plasma catalysis. Three different pore shapes are considered, i.e., a conical pore with maximum diameter of $50 \mu \mathrm{m}$ and small opening of $5 \mu \mathrm{m}$, a cylindrical pore with constant diameter of $50 \mu \mathrm{m}$, and a conical pore with large opening of $50 \mu \mathrm{m}$ and narrow bottom diameter of $5 \mu \mathrm{m}$. In all cases, the pore depth is kept constant at $100 \mu \mathrm{m}$. 
The results indicate that for a conical pore with small opening at time phase $\varphi=0$ and $\varphi=$ $0.5 \pi$, the electric field near and inside the pore is directed downwards, and it is significantly enhanced at the pore opening, due to the small size there. Therefore, the ionization mainly takes place at the pore opening. However, the ionization rate at $\varphi=0.5 \pi$ is much lower than at $\varphi=0$, due to the lower electron density near the bottom dielectric at this condition. As the time phase increases to $\pi$ and $1.5 \pi$, the electric field at the bottom of the pore is enhanced, and it is directed upwards, attracting the electrons towards the bottom. Thus, the ionization mainly takes place near the bottom of the pore, but the absolute value is lower, because the pore opening is too small, limiting the electron flow into the pore.

For a cylindrical pore, the electrical behavior and the corresponding ionization rate profiles are clearly different. At $\varphi=0$, the electric field is not only enhanced at the corners near the opening but also at the bottom of the pore, and the value inside the pore/is only slightly lower. The ionization rate is thus characterized by a prominent peak at the pore opening, due to the strong electric field inside the pore and the high electron density above the pore. At $\varphi=0.5 \pi$, the weaker electric field and the lower electron density are responsible for the negligible ionization rate. At a later time, i.e., $\varphi=\pi$, the electric field is significantly enhanced at the bottom of the pore, and it is directed upwards, effectively heating the electrons and attracting them towards the bottom. This gives rise to a pronounced ionization inside the pore. At $\varphi=$ $1.5 \pi$, the electric field distribution looks the same, but the electron density is lower, so the ionization rate exhibits a similar profile, but the absolute value is more than one order of magnitude lower.

Finally, in a conical pore with large opening, the electric field reaches its maximum at the bottom of the pore, throughout the entire cycle of the applied voltage. However, the electric field increases gradually from the top of the pore to the bottom at $\varphi=0$ and $\varphi=0.5 \pi$, whereas an abrupt increase near the pore bottom is observed at $\varphi=\pi$ and $\varphi=1.5 \pi$. Therefore, a broad maximum of the ionization rate is observed inside and above the pore at $\varphi=0$, while it is characterized by a sharp peak at the bottom of the pore at $\varphi=\pi$. At $\varphi=0.5 \pi$ and $\varphi=1.5 \pi$, the ionization rate is again verylow, due to the low electron density in these cases.

In conclusion, for a conical pore with small opening, the time-averaged electric field is remarkably enhanced at the opening and the bottom corners of the pore, due to the tip-like structure there. Besides, the electric field inside the pore is still significant, giving rise to prominent ionization throughout the pore. For a cylindrical pore, the time-averaged electric field is only enhanced at the bottom of the pore, with a much lower value, and hence this 
limits the plasma generation inside the pore. Finally, for a conical pore with large opening, the ionization rate exhibits a significant peak at the bottom of the pore, due to the strongest electric field there. It would be good to validate our simulations with experiments, but to our knowledge, no experimental data are available to be compared with these calculation results. However, in view of the increasing interest in plasma catalysis, we hope that such experiments can be performed in the future.

Overall, the electric field enhancement, and consequently also the ionization rate, and thus the plasma generation, is most pronounced for the conical pores with either small or large opening. The results obtained in this work help us to obtain more insight in the electric field enhancement, and thus the modified plasma generation, caused by various pore shapes, which is of utmost importance for plasma catalysis. Considering the geometry, the discharge inside catalyst pores is similar to micro hollow cathode discharges. The electrons are accelerated by the strong electric field inside the pore or cathode, and they collide with the gas, leading to plasma generation. Therefore, the obtained results might also be very interesting for the investigation of micro-discharges, micro hollow cathode discharges, etc.

\section{Acknowledgments}

This work was supported by the Fund for Scientific Research Flanders (FWO) (Grant No. G.0217.14N) and the Fundamental Research Funds for the Central Universities (Grant No. DUT17LK52).

\section{References}

[1] Chen H L, Lee H M, Chen S H, Chao Y and Chang M B 2008 Appl. Catal. B: Environ. 851

[2] Van Durme J, Dewulf J, Leys C and Van Langenhove H 2008 Appl. Catal. B: Environ. 78324

[3] Whitehead J C 2010 Pure Appl. Chem. 821329

[4] Kim H H, Teramoto Y, Ogata A, Takagi H and Nanba T 2016 Plasma Chem. Plasma Process. 3645

[5] Chen H L, Lee H M, Chen S H, Chang M B, Yu S J and Li S N 2009 Env. Sci. Technol. 432216

[6] Neyts E C and Bogaerts A 2014 J. Phys. D: Appl. Phys. 47224010 
[7] Kim H H, Teramoto Y, Negishi N and Ogata A 2015 Catal. Today 25613

[8] Neyts E C 2016 Plasma Chem. Plasma Process. 36185

[9] Whitehead J C 2016 J. Phys. D: Appl. Phys. 49243001

[10] Neyts E C, Ostrikov K, Sunkara M K and Bogaerts A 2015 Chem. Rev. 11513408

[11] Patil B S, Cherkasov N, Lang J, Ibhadon A O, Hessel V and Wang Q 2016 Appl. Catal. B: Environ. 194123

[12] Witvrouwen T, Paulussen S and Sels B 2012 Plasma Processes Polym. 9750

[13] Liu C J, Vissokov G P and Jang B W L 2002 Catal. Today 72173

[14] Tang X, Li K, Yi H, Ning P, Xiang Y, Wang J and Wang C 2012 J. Phys. Chem. C 116 10017

[15] Tu X, Gallon H J and Whitehead J C 2013 Catal. Today 211120

[16] Demidyuk V and Whitehead J C 2007 Plasma Chem. Plasma Process. 2785

[17] Nozaki T and Okazaki K 2013 Catal. Today 21129

[18] Ogata A, Yamanouchi K, Mizuno K, Kushiyama S and Yamamoto T 1999 IEEE Trans. Ind. Appl. 351289

[19] Roland U, Holzer F and Kopinke F D 2002 Catal. Today 73315

[20] Tu X, Gallon H J and Whitehead J C 2011 J. Phys. D: Appl. Phys. 44482003

[21] Takuma T 1991 IEEE Trans. Electr. Insul. 26500

[22] Chen H L, Lee H M, Chen S H and Chang M B 2008 Ind. Eng. Chem. Res. 472122

[23] Fridman A 2012 Plasma chemistry. Cambridge University Press, New York

[24] Zhang Y R, Van Laer K, Neyts E C and Bogaerts A 2016 Appl. Catal. B: Environ. 185 56

[25] Stollenwerk L 2009 New J. Phys. 11103034

[26] Wagner H E, Brandenburg R, Kozlov K V, Sonnenfeld A, Michel P and Behnke J F 2003 Vacuum 71 417-436

[27] Wang M, Foster J E and Kushner M J 2011 IEEE Trans. Plasma Sci. 392244

[28] Xiong Z and Kushner M J 2012 Plasma Sources Sci. Technol. 21034001

[29] Van Laer K and Bogaerts A 2016 Plasma Sources Sci. Technol. 25015002

[30] Takaki K, Urashima K and Chang J S 2004 IEEE Trans. Plasma Sci. 322175

[31] Yu Q, Kong M, Liu T, Fei J and Zheng X 2012 Plasma Chem. Plasma Process. 32153

[32] Comsol Website; http://www.comsol.com

[33] Hagelaar G J M and Pitchford L C 2005 Plasma Sources Sci. Technol. 14722

[34] Morgan database, www.lxcat.net, retrieved on July 25, 2013.

[35] Deloche R, Monchicourt P, Cheret M and Lambert F 1976 Phys. Rev. A 131140 
[36] Wang Q, Economou D J and Donnelly V M 2006 J. Appl. Phys. 100023301

[37] Emmert F, Angermann H H, Dux R and Langhoff H 1988 J. Phys. D: Appl. Phys. 21667

[38] Alves L L, Gousset G and Ferreira C M 1992 J. Phys. D: Appl. Phys. 251713

[39] Carbone E, Schregel C and Czarnetzki U 2016 Plasma Sources Sci. Technol. 25054004

[40] Edgcombe C J and Valdre U 2001 J. Microsc. 203188

[41] Bhoj A N and Kushner M J 2006 J. Phys. D: Appl. Phys. 391594

[42] Bhoj A N and Kushner M J 2008 Plasma Sources Sci. Technol. 17035024

[43] Bhoj A N and Kushner M J 2008 Plasma Sources Sci. Technol. 17035025

[44] Schregel C, Carbone E, Luggenholscher D and Czarnetzki U 2016 Plasma Sources Sci. Technol. 25054003

\section{Figure Captions}

Figure 1 Time-averaged ionization rate profiles for three different pore shapes. The maximum pore diameter (i.e., at the bottom in (a), constant in (b) and at the opening in (c)) is fixed at 50 $\mu \mathrm{m}$, while the minimum pore diameter (i.e., at the opening in (a) and at the bottom in (c)) is 5 $\mu \mathrm{m}$. The pore depth is $100 \mu \mathrm{m}$ in all cases. Note that the same color scale is used for the three pore shapes, to allow easy comparison, but the minimum and maximum values of the ionization rate are in each case indicated above and below the color legend.

Figure 2 Ionization rate along the centerline of the pore (indicated by a vertical line in the inset illustrations), inside and above the pore, at four different times within one cycle of the applied voltage (see inset in (a)), for three different pore shapes, as in figure 1.

Figure 3 Electric field profiles at four different times within one cycle of the applied voltage, for a conical pore with small opening. The normalized vectors indicate the direction of the electric field. Note that the same color scale is used for the four time phases, to allow easy comparison, but the minimum and maximum values of the electric field are in each case indicated above and below the color legend.

Figure 4 Electron density profiles at four different times within one cycle of the applied voltage, for a conical pore with small opening. The vectors indicate the direction and magnitude of the electron flux. Note that a different color scale is used for the four time phases, to clearly see the electron density profiles in each case. 
Figure 5 Electric field profiles at four different times within one cycle of the applied voltage, for a cylindrical pore. The normalized vectors indicate the direction of the electric field. Note that the same color scale is used for the four time phases, to allow easy comparison, but the minimum and maximum values of the electric field are in each case indicated above and below the color legend.

Figure 6 Electron density profiles at four different times within one cycle of the applied voltage, for a cylindrical pore. The vectors indicate the direction and magnitude of the electron flux. Note that a different color scale is used for the four time phases, to clearly see the electron density profiles in each case.

Figure 7 Electric field profiles at four different times within one cycle of the applied voltage, for a conical pore with large opening. The normalized vectors indicate the direction of the electric field. Note that the same color scale is used for the four time phases, to allow easy comparison, but the minimum and maximum values of the electric field are in each case indicated above and below the color legend.

Figure 8 Electron density profiles at four different times within one cycle of the applied voltage, for a conical pore with large opening. The vectors indicate the direction and magnitude of the electron flux. Note that a different color scale is used for the four time phases, to clearly see the electron density profiles in each case.

Figure 9 Time-averaged electric field profiles for three different pore shapes, as in figure 1. The normalized vectors indicate the direction of the electric field. Note that the same color scale is used for the three pore shapes, to allow easy comparison, but the minimum and maximum values of the electric field are in each case indicated above and below the color legend.

Figure 10 Time-averaged electron temperature profiles for three different pore shapes, as in figure 1 . Note that the same color scale is used for the three pore shapes, to allow easy comparison, but the minimum and maximum values of the electron temperature are in each case indicated above and below the color legend. 
Figure 11 Time-averaged electron density profiles for three different pore shapes, as in figure 1. The vectors indicate the direction and magnitude of the electron flux. Note that the same color scale is used for the three pore shapes, to allow easy comparison, but the minimum and maximum values of the electron density are in each case indicated above and below the color legend.

Figure 12 Time-averaged $\mathrm{He}^{\mathrm{M}}$ density profiles for three different pore shapes, as in figure 1 . Note that the same color scale is used for the three pore shapes, to allow easy comparison, but the minimum and maximum values of the $\mathrm{He}^{\mathrm{M}}$ density are in each case indicated above and below the color legend.

Figure 13 Time-averaged $\mathrm{He}_{2}{ }^{\mathrm{M}}$ density profiles for three different pore shapes, as in figure 1 . Note that the same color scale is used for the three pore shapes, to allow easy comparison, but the minimum and maximum values of the $\mathrm{He}_{2}{ }^{\mathrm{M}}$ density are in each case indicated above and below the color legend.

Figure 14 Time-averaged electron density, $\mathrm{He}^{\mathrm{M}}$ density and $\mathrm{He}_{2}{ }^{\mathrm{M}}$ density, along the vertical centerline for a cylindrical pore.

Figure 15 Time-averaged surface charge density at the dielectric surface for three different pore shapes, as in figure 1. 

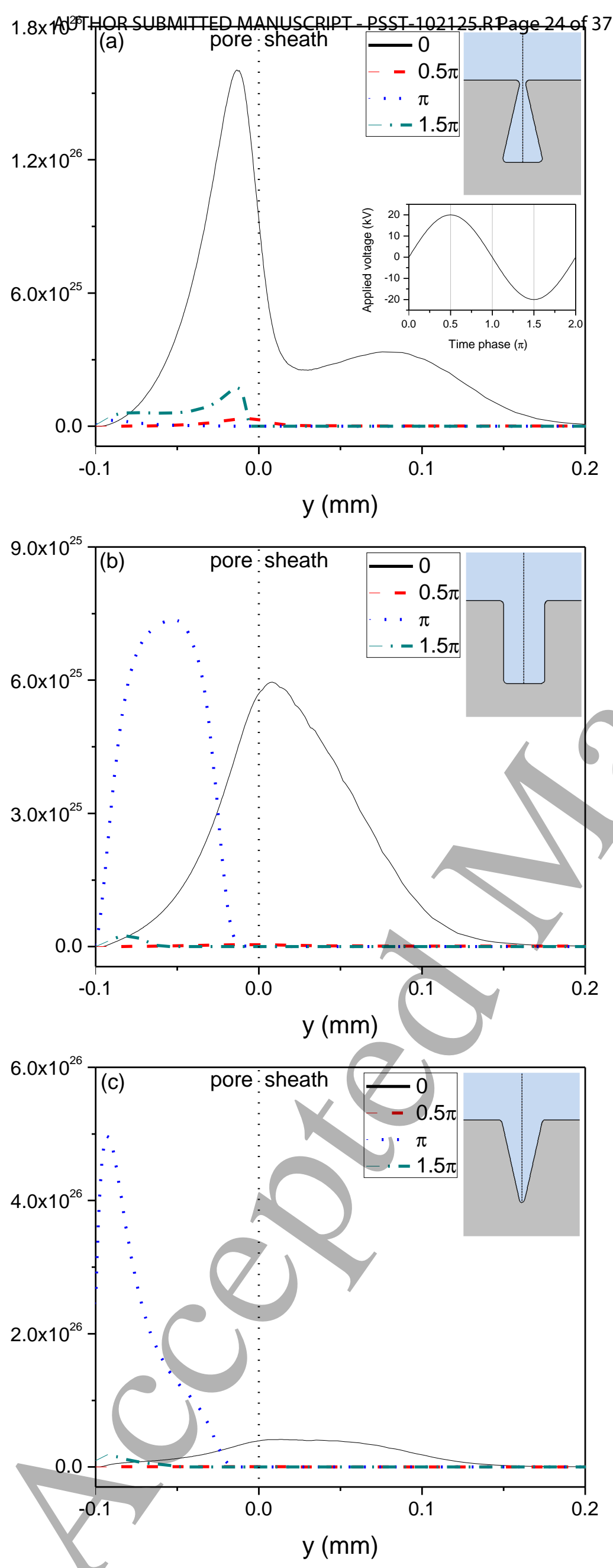

Figure 2 
(a) 0

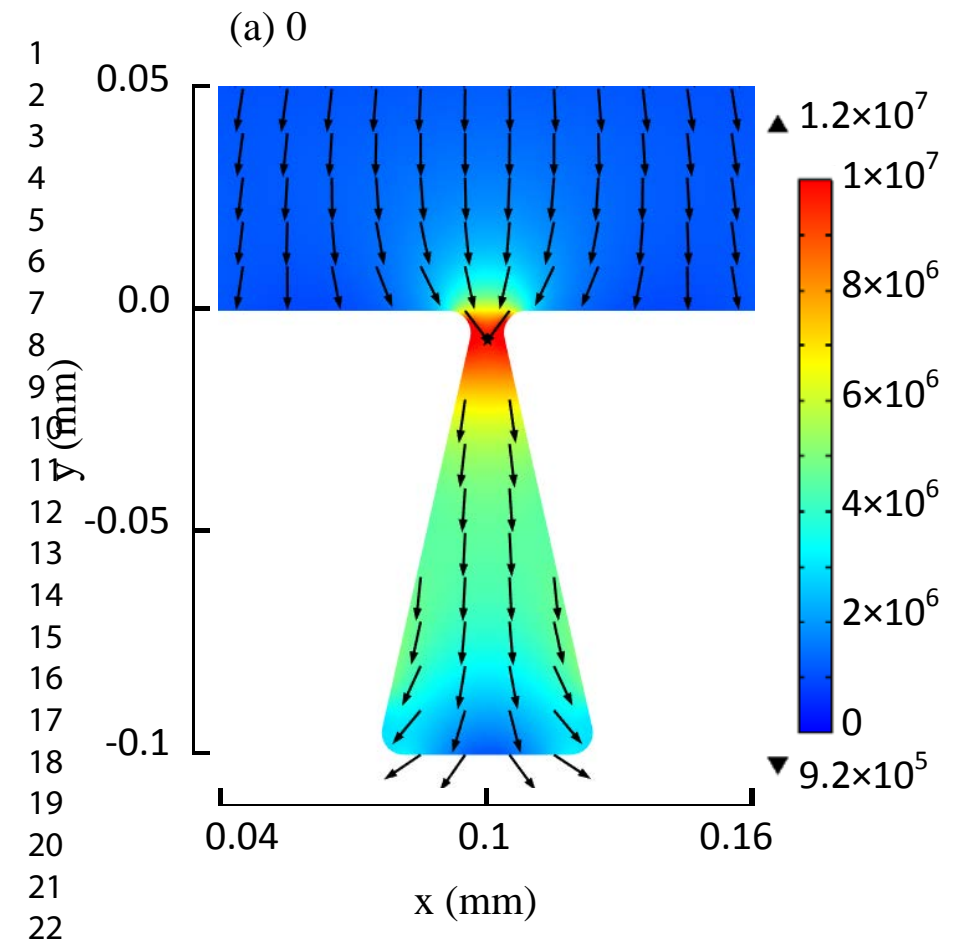

(c) $\pi$

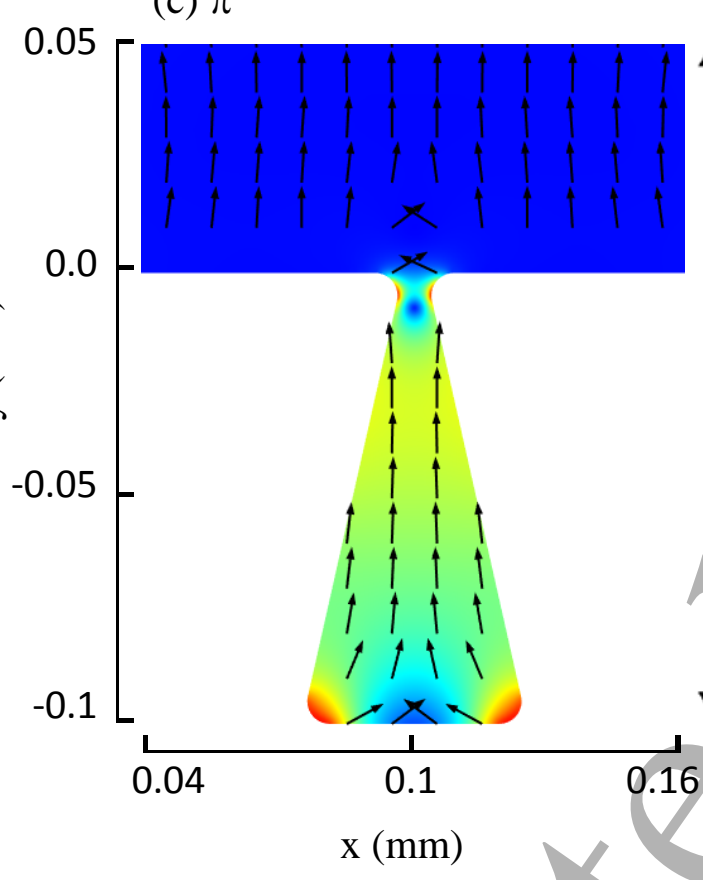

(b) $0.5 \pi$

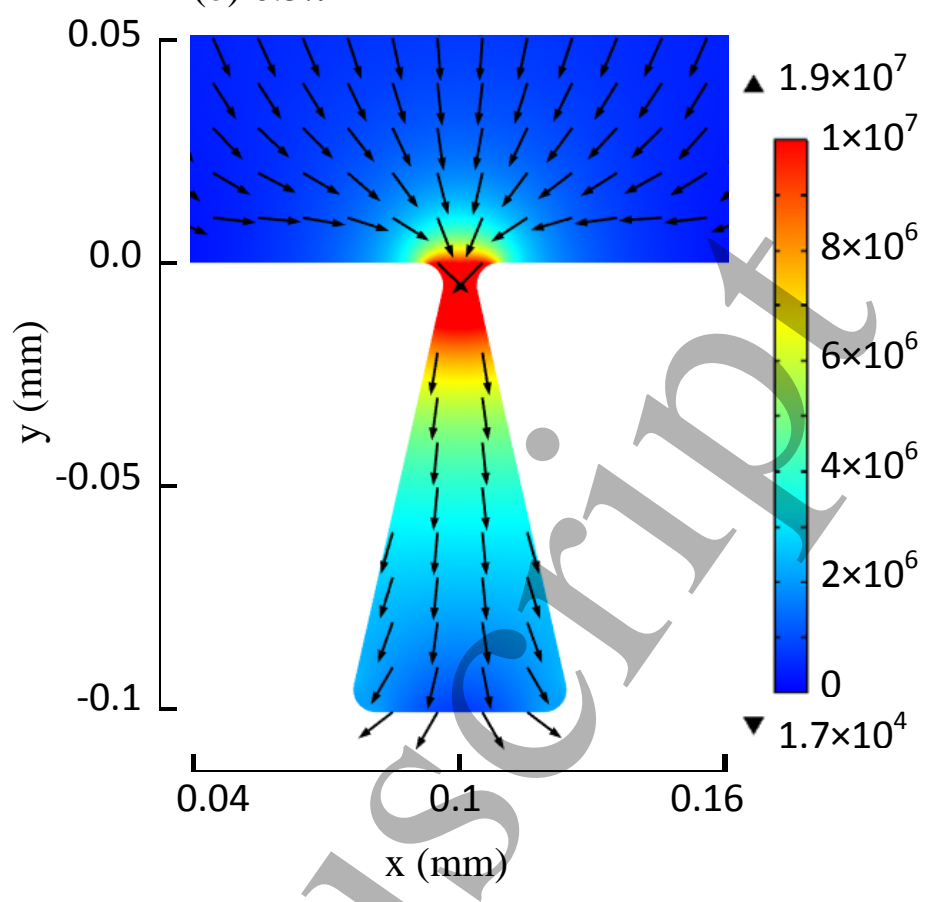

(d) $1.5 \pi$

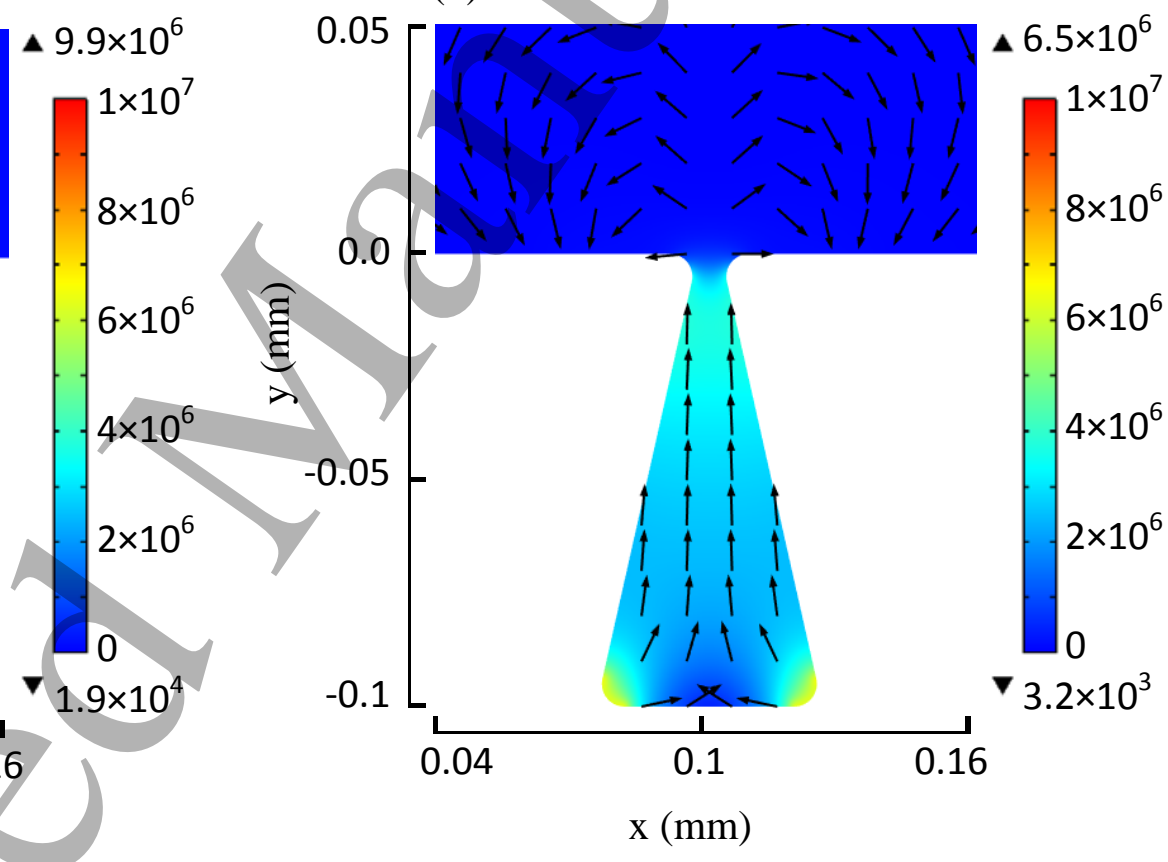

Figure 3 
(a) 0

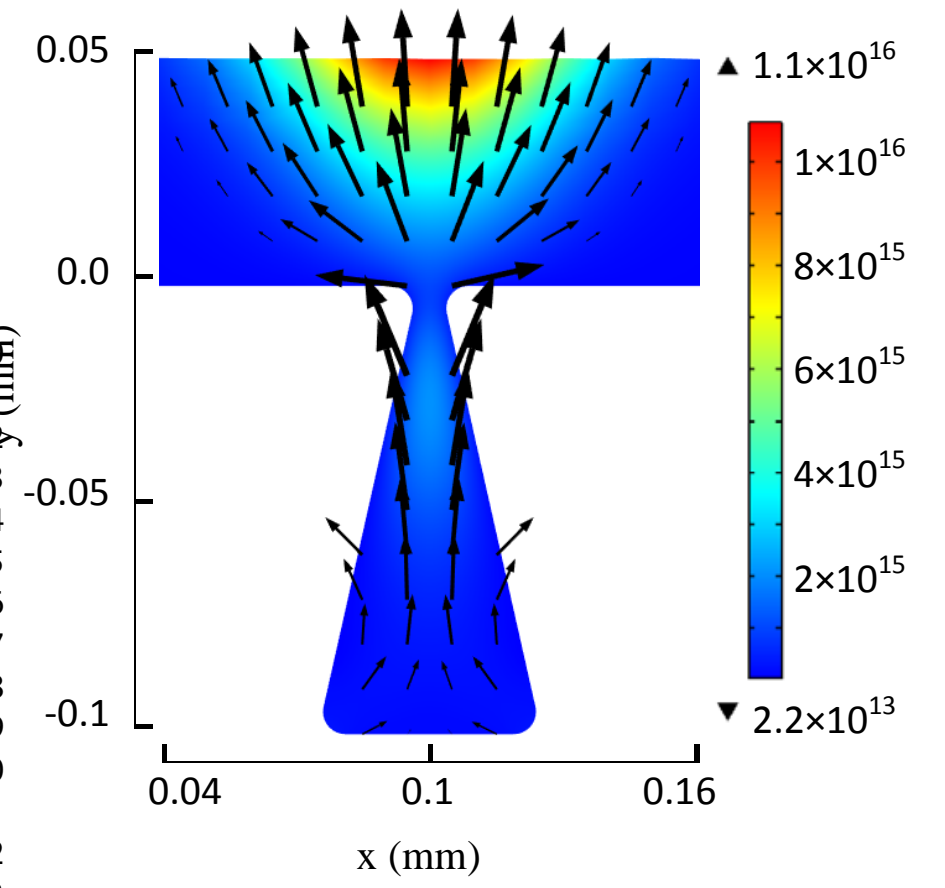

(c) $\pi$

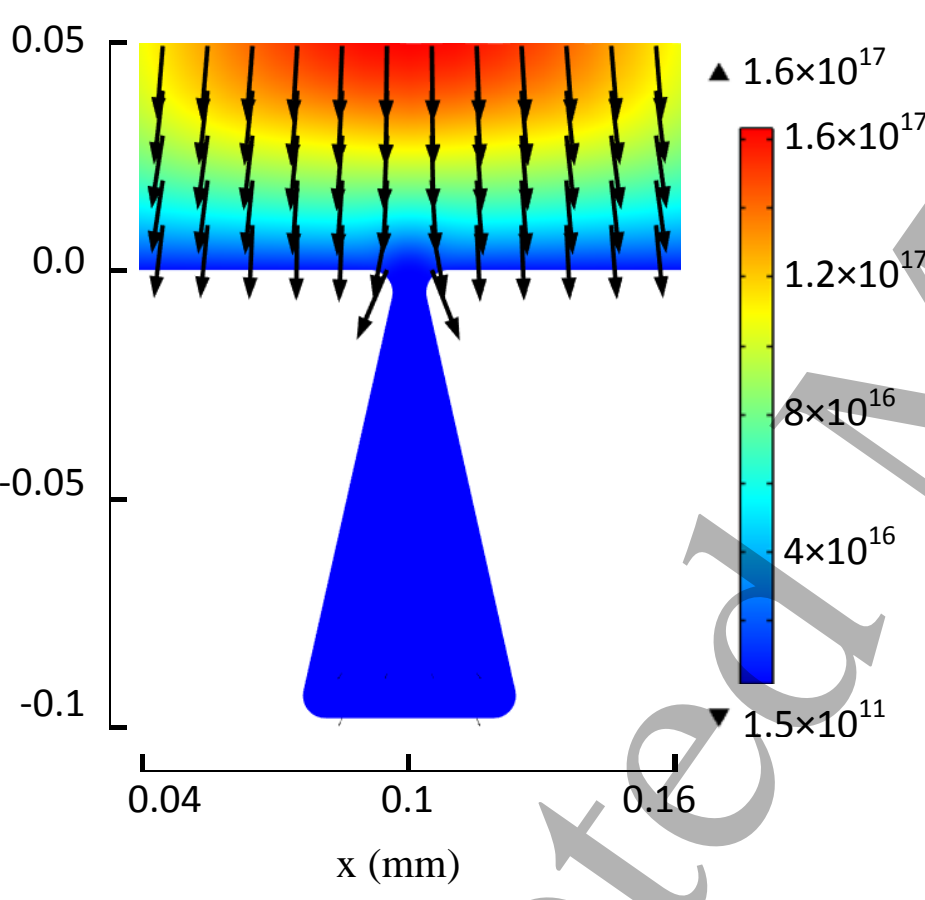

(b) $0.5 \pi$

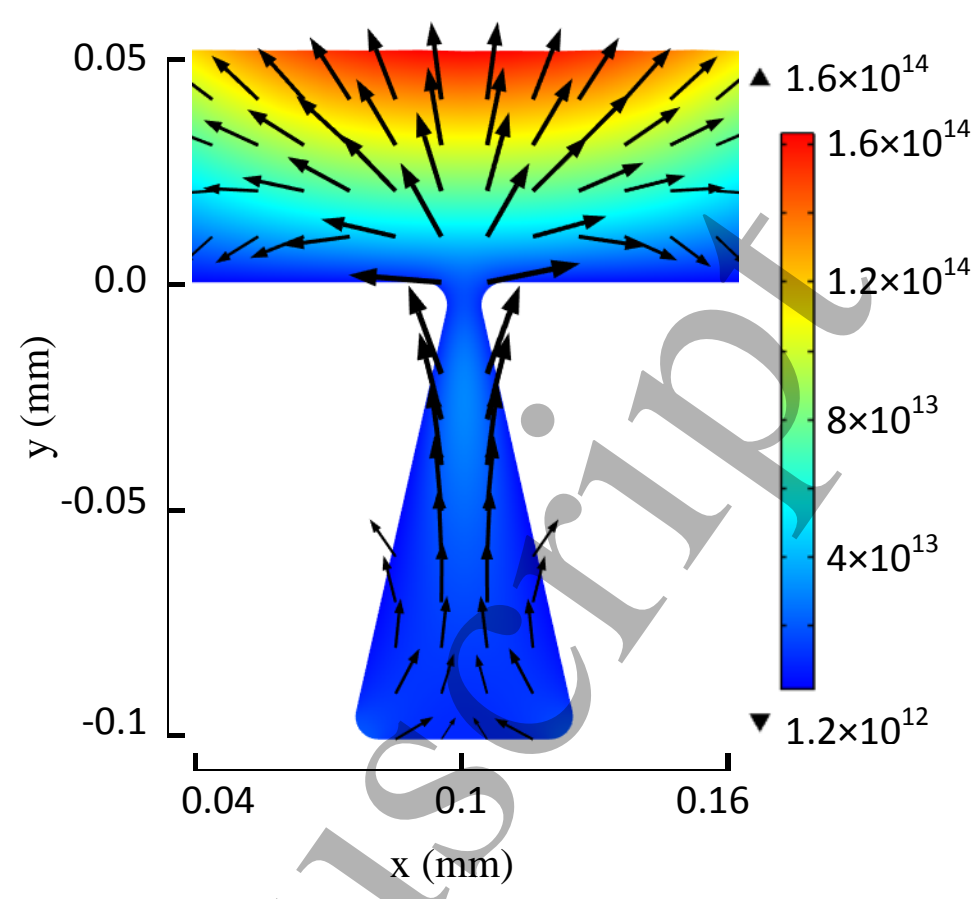

(d) $1.5 \pi$

Figure 4 
(a) 0

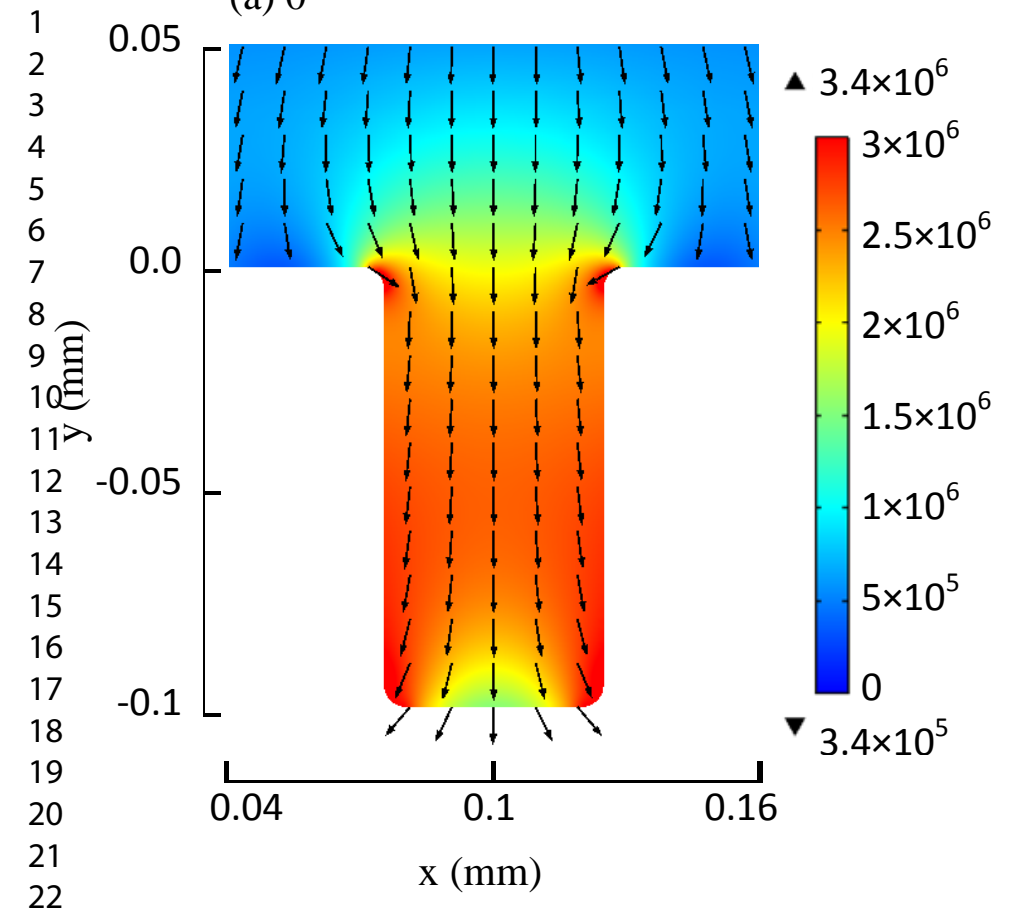

(c) $\pi$

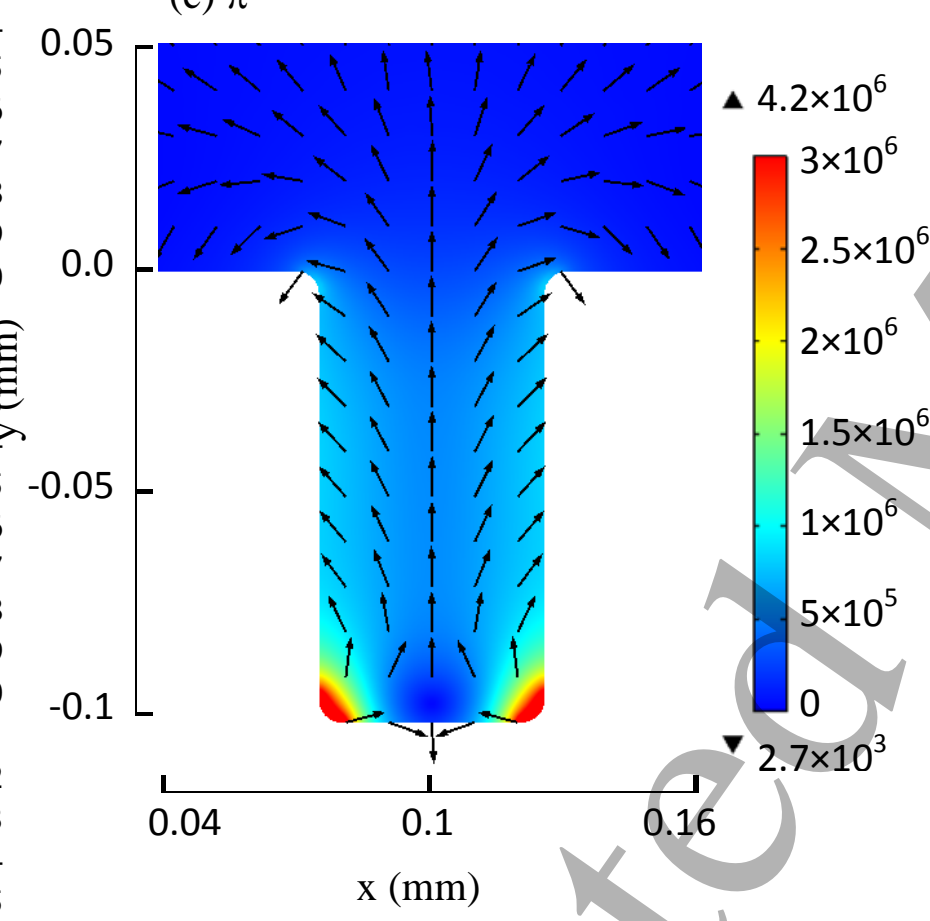

(b) $0.5 \pi$

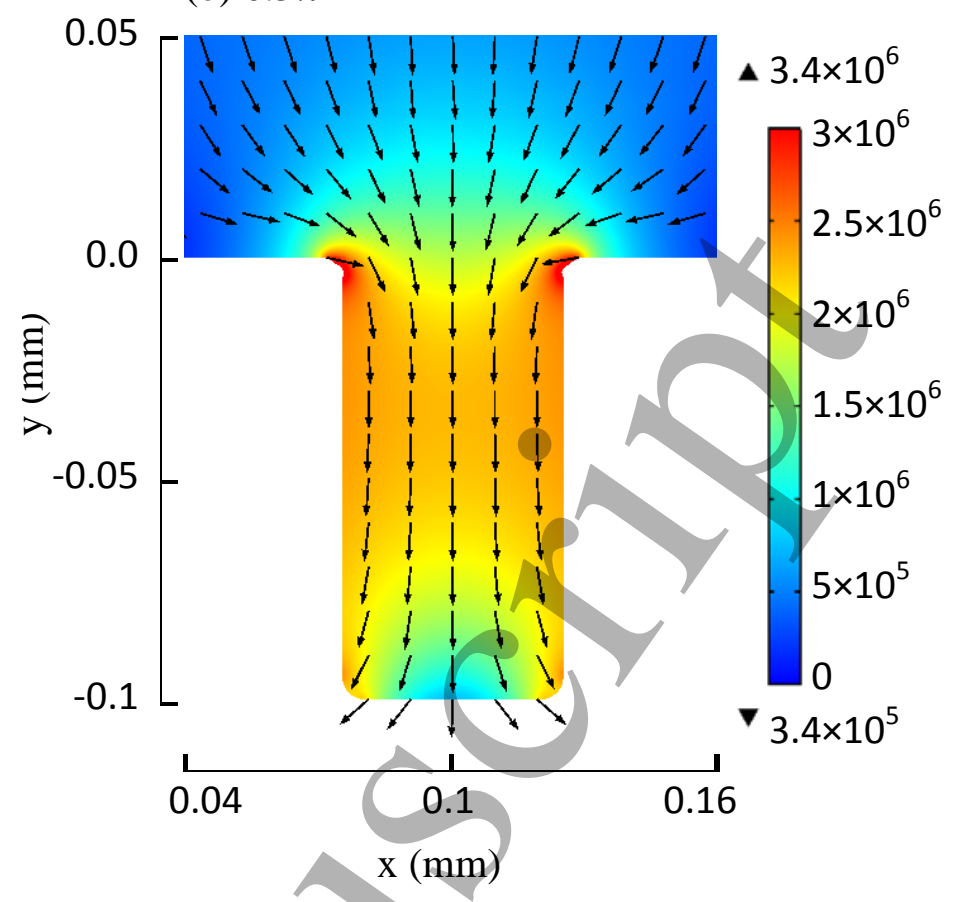

(d) $1.5 \pi$

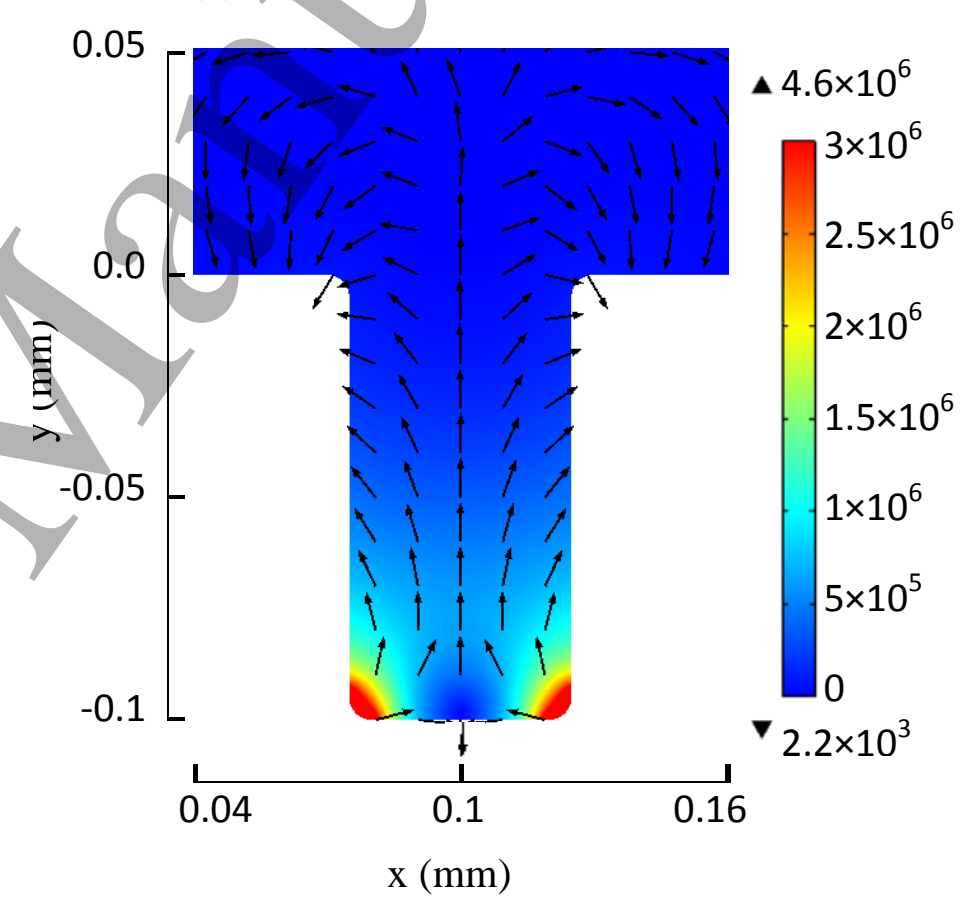

Figure 5 
(a) 0

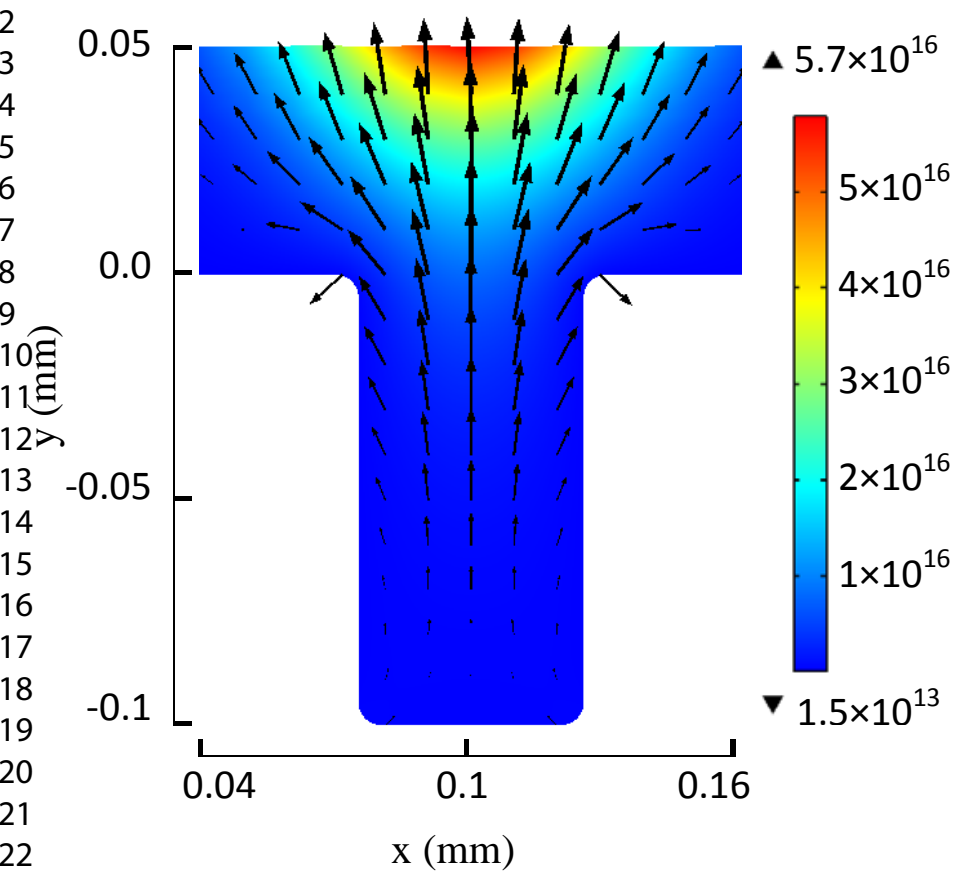

(c) $\pi$

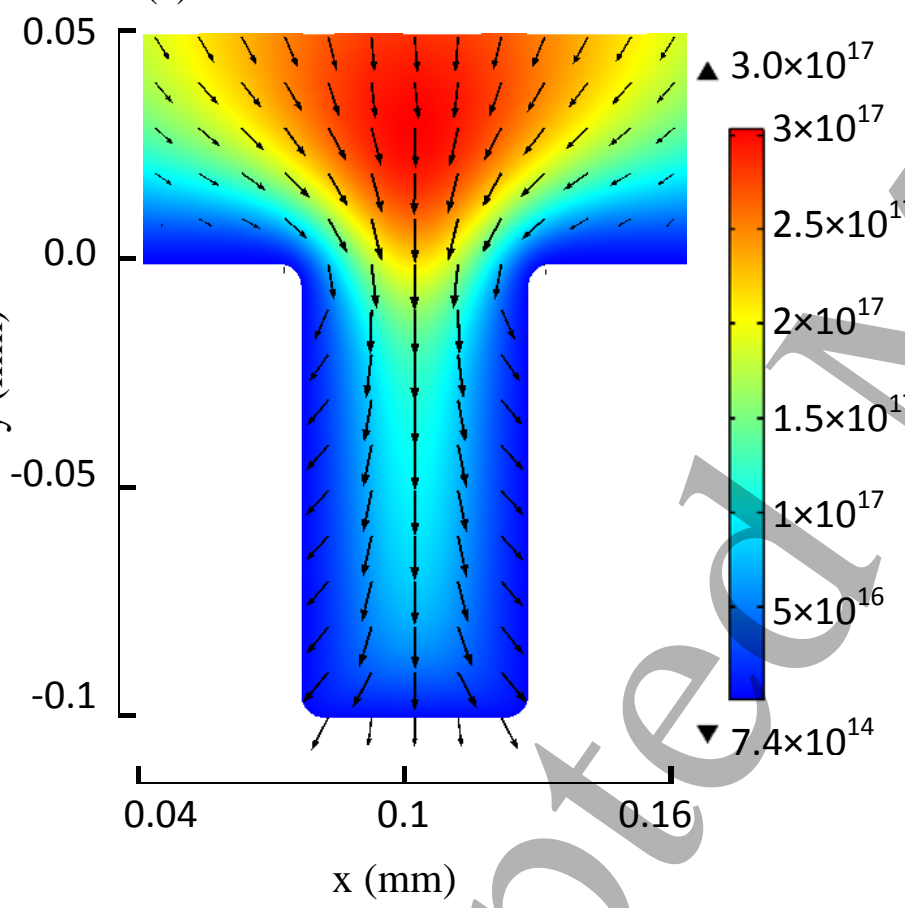

(b) $0.5 \pi$

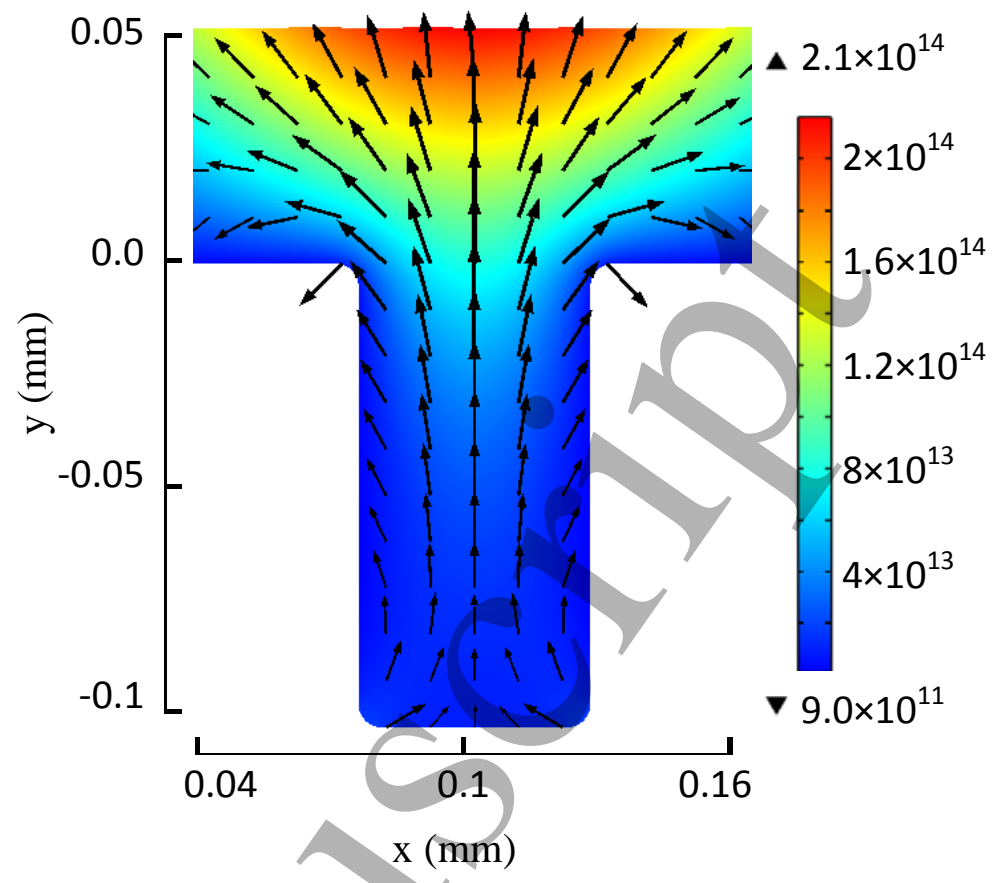

(d) $1.5 \pi$
24

25

27

28

29

30

31

33

34

35 E

$36>$

37

40

41

42

48

49

50

51

52

53

54

55

56

57

58

59

60

Figure 6 
(a) 0

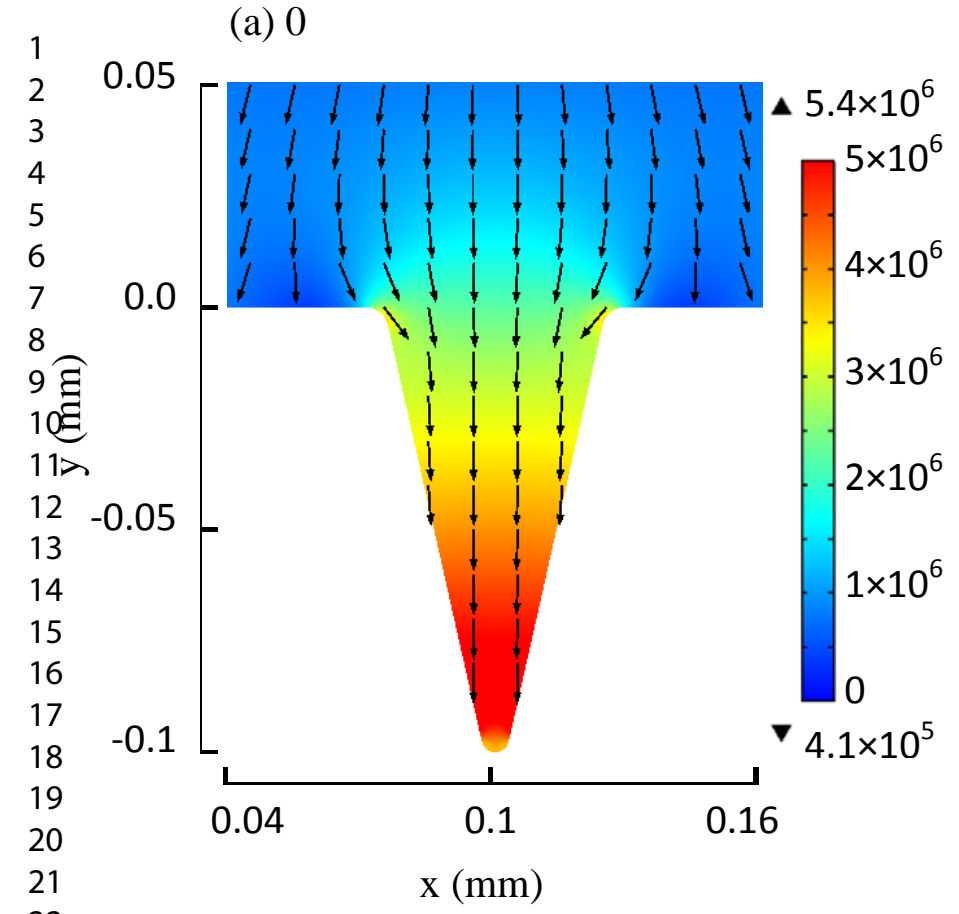

(c) $\pi$

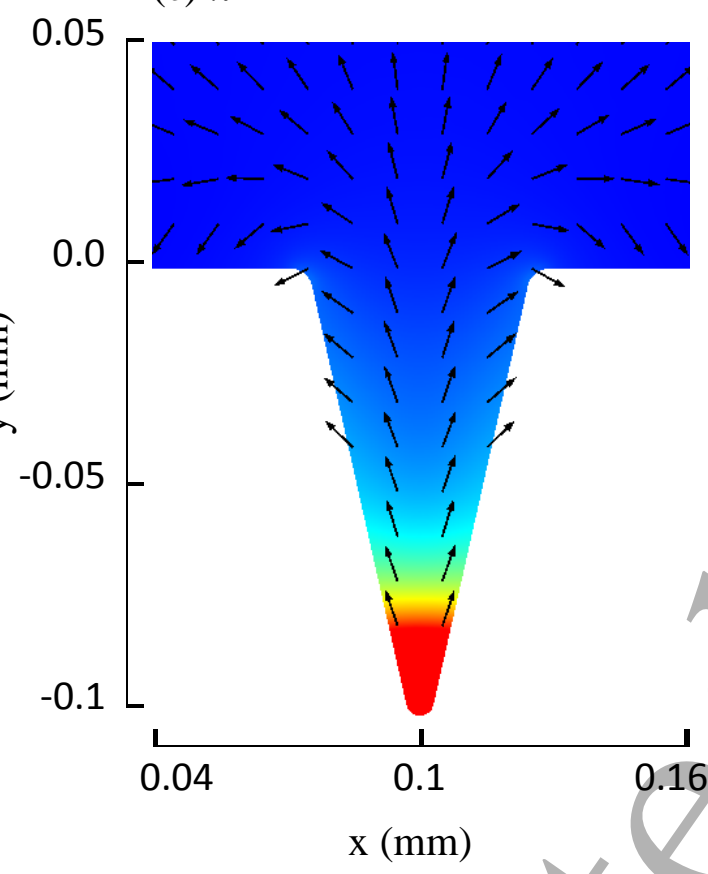

(b) $0.5 \pi$

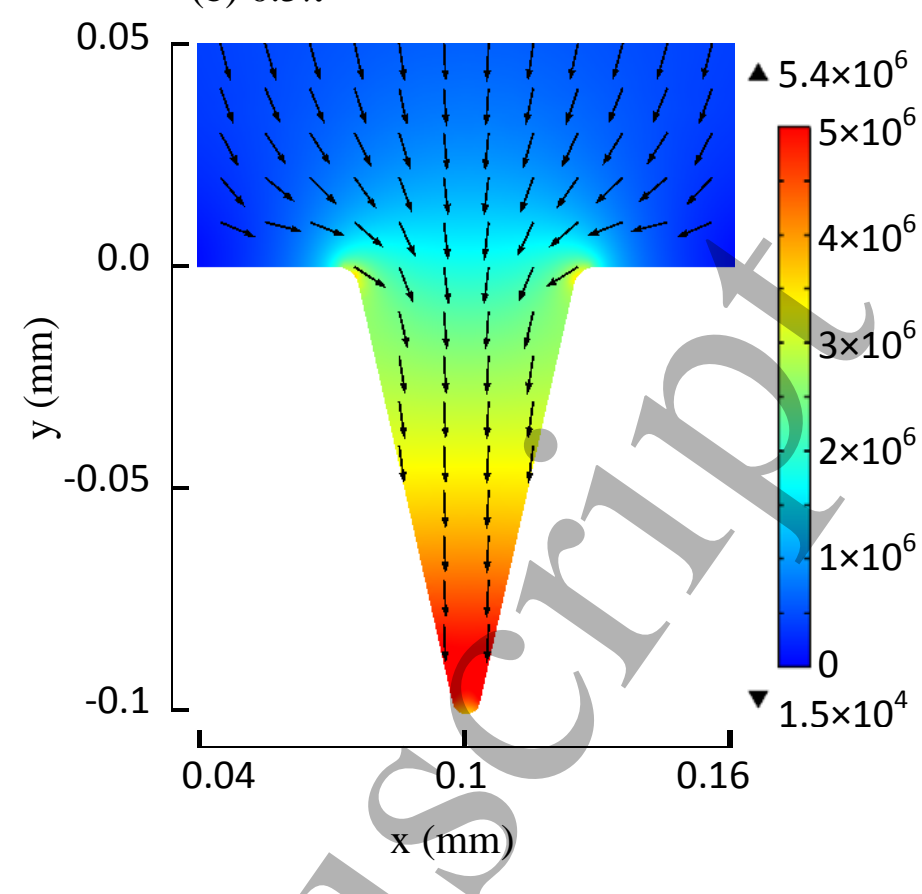

(d) $1.5 \pi$

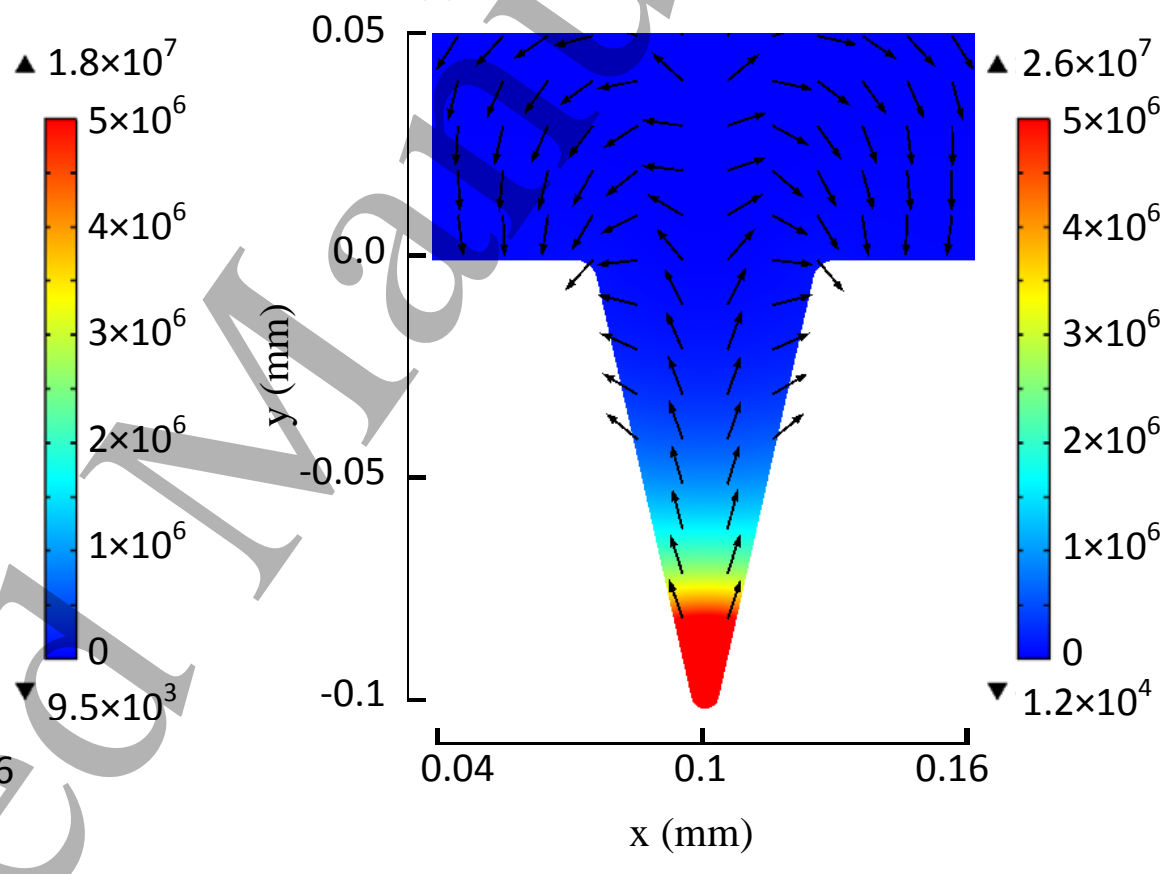

Figure 7 
(a) 0

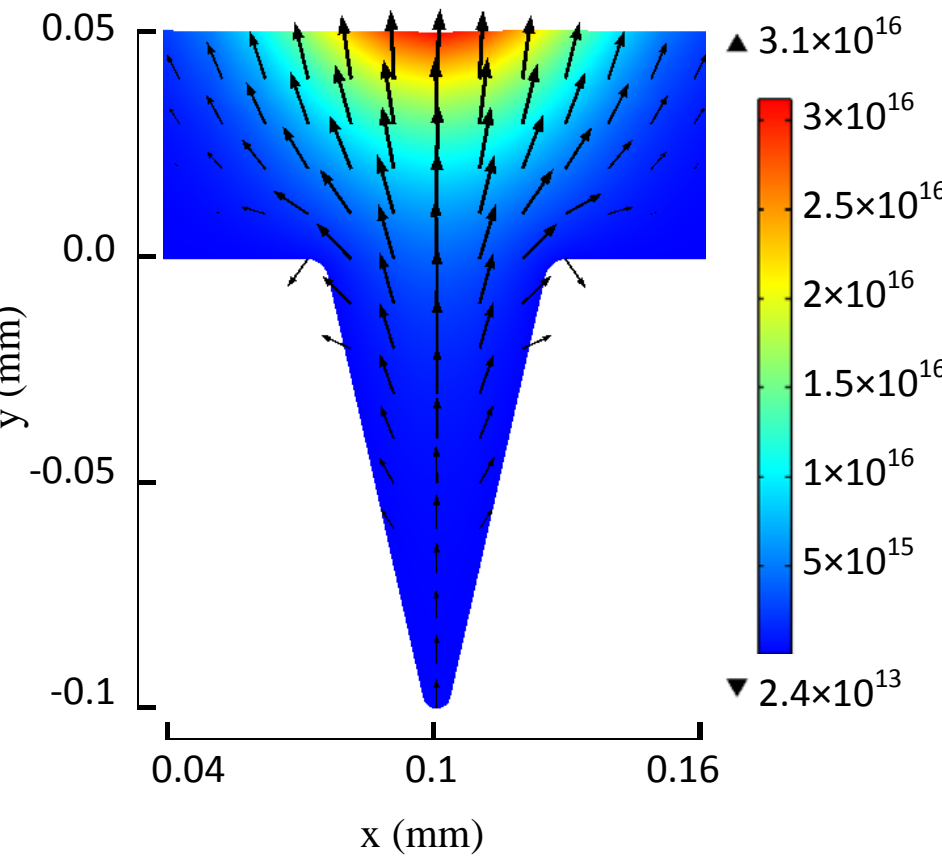

(c) $\pi$

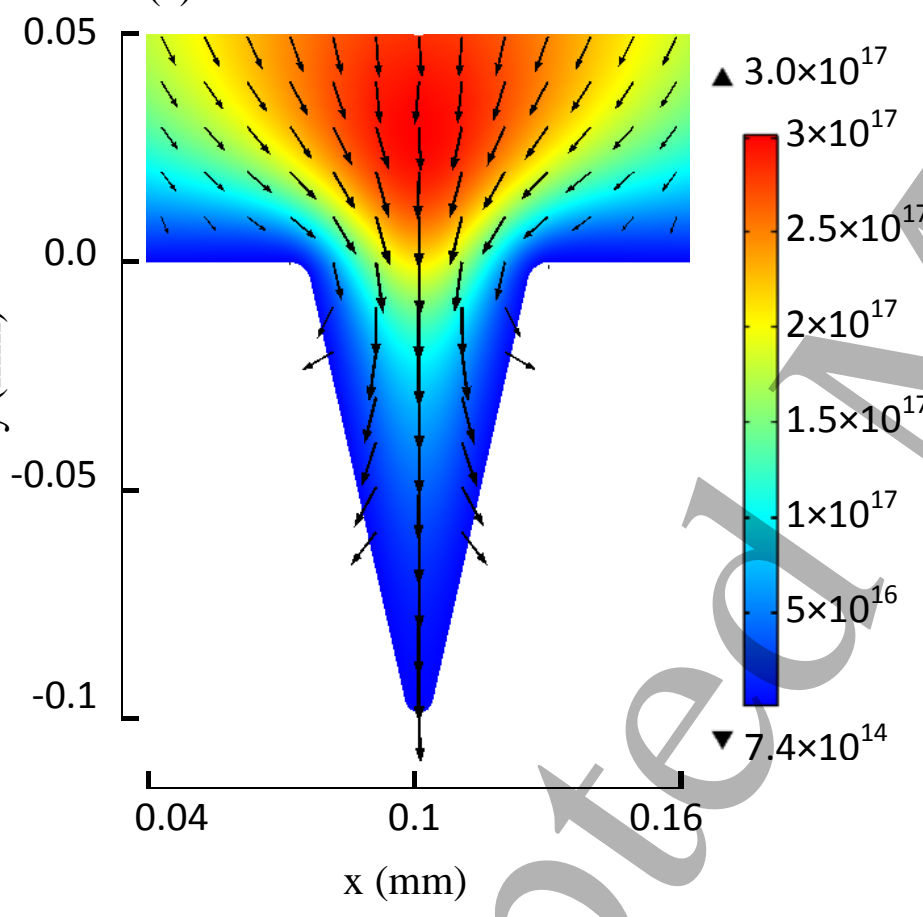

(b) $0.5 \pi$

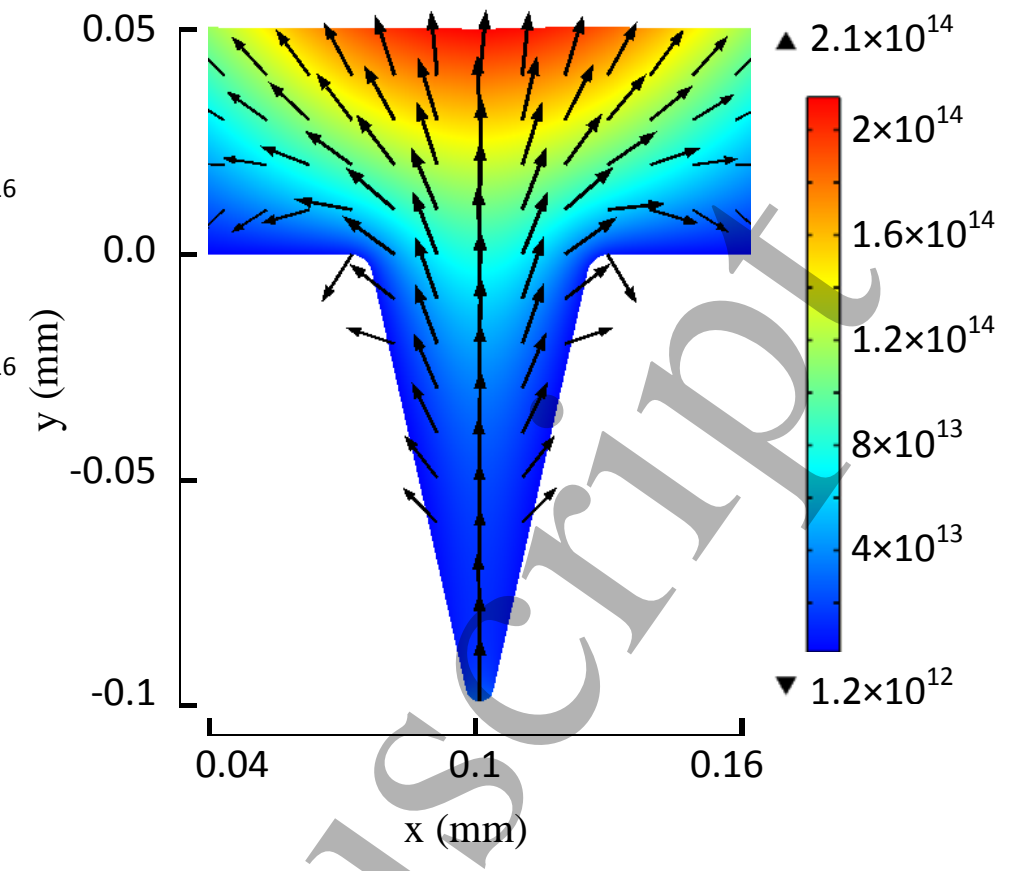

(d) $1.5 \pi$

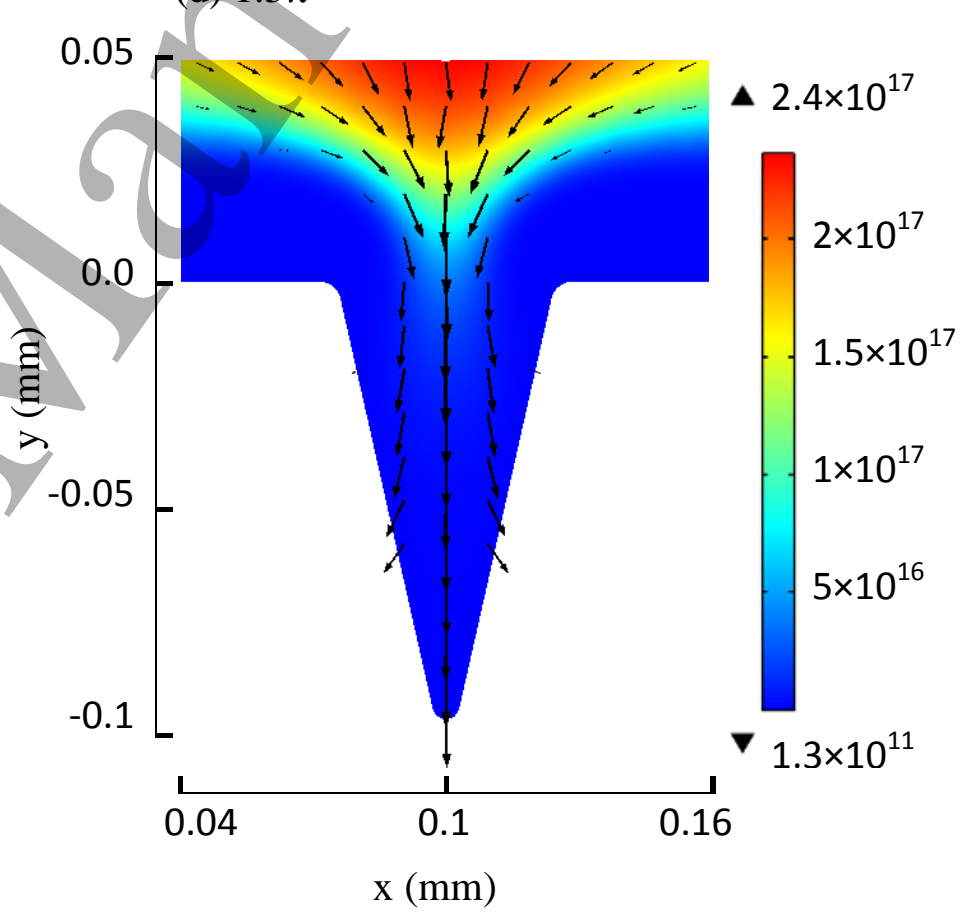

Figure 8 
(a)

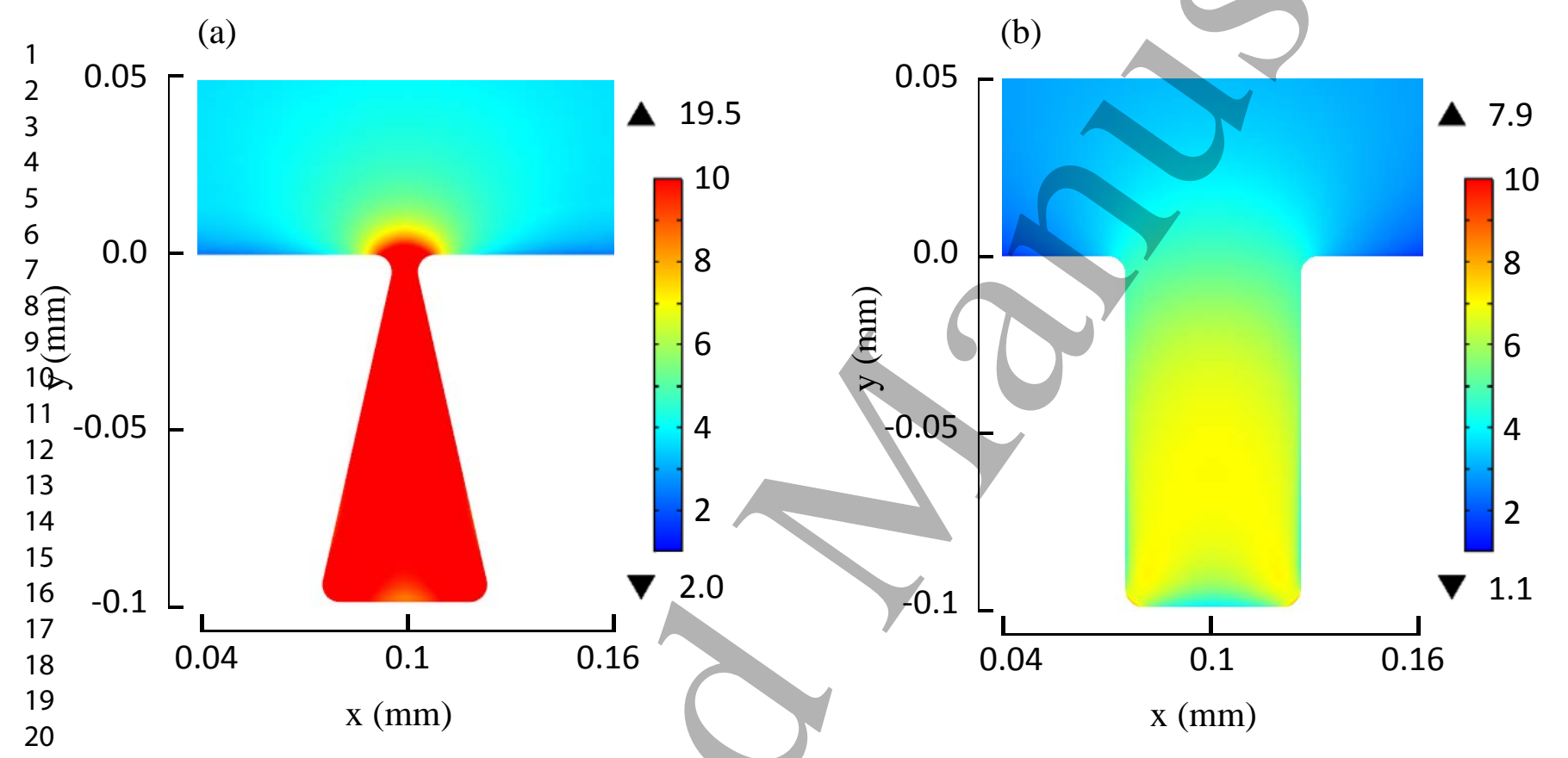

(c)

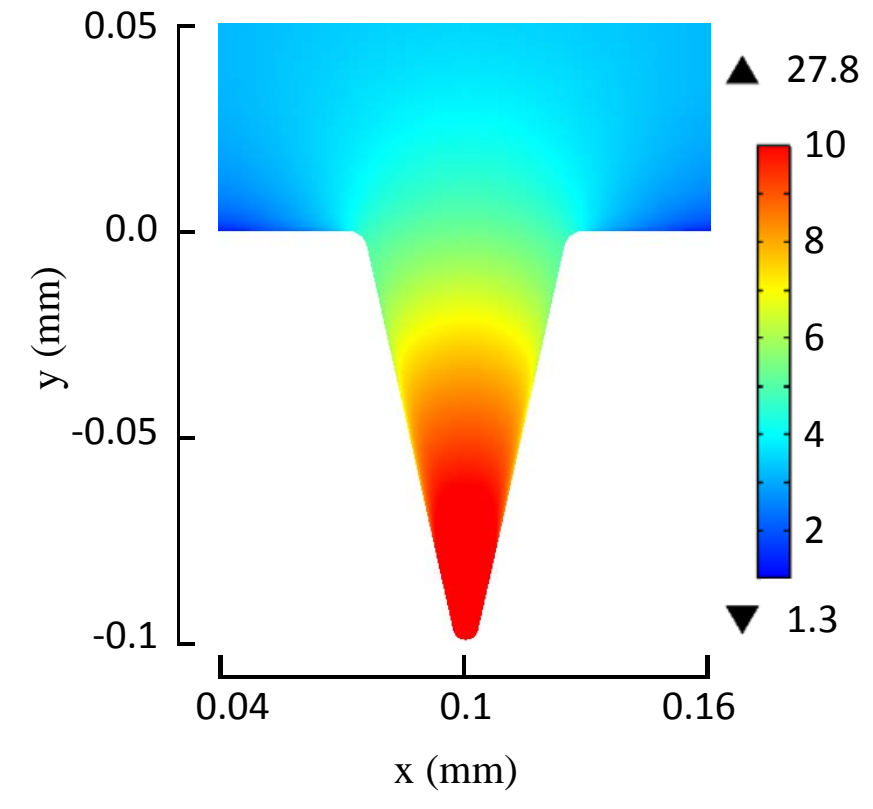

Figure 10 
(a)

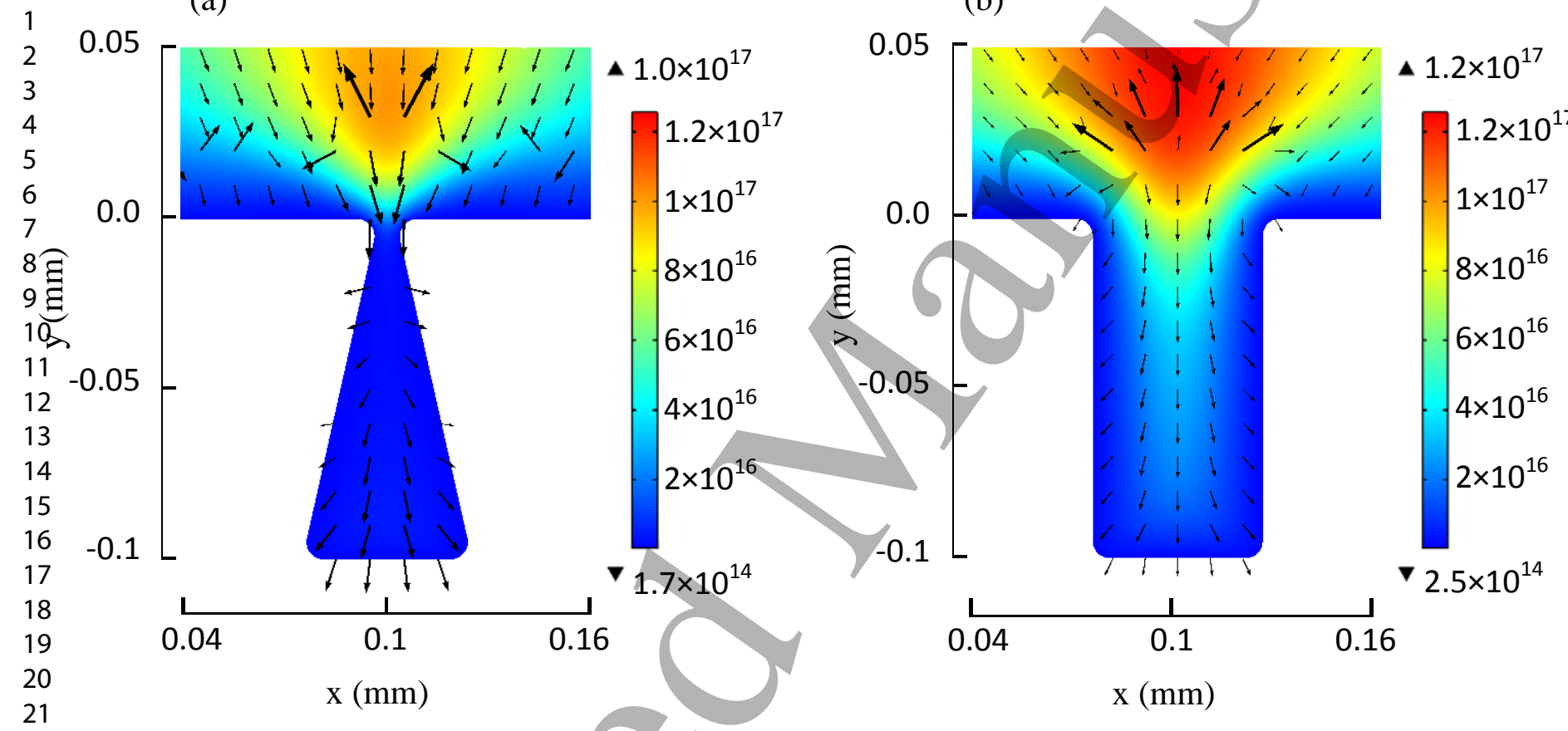

(c)

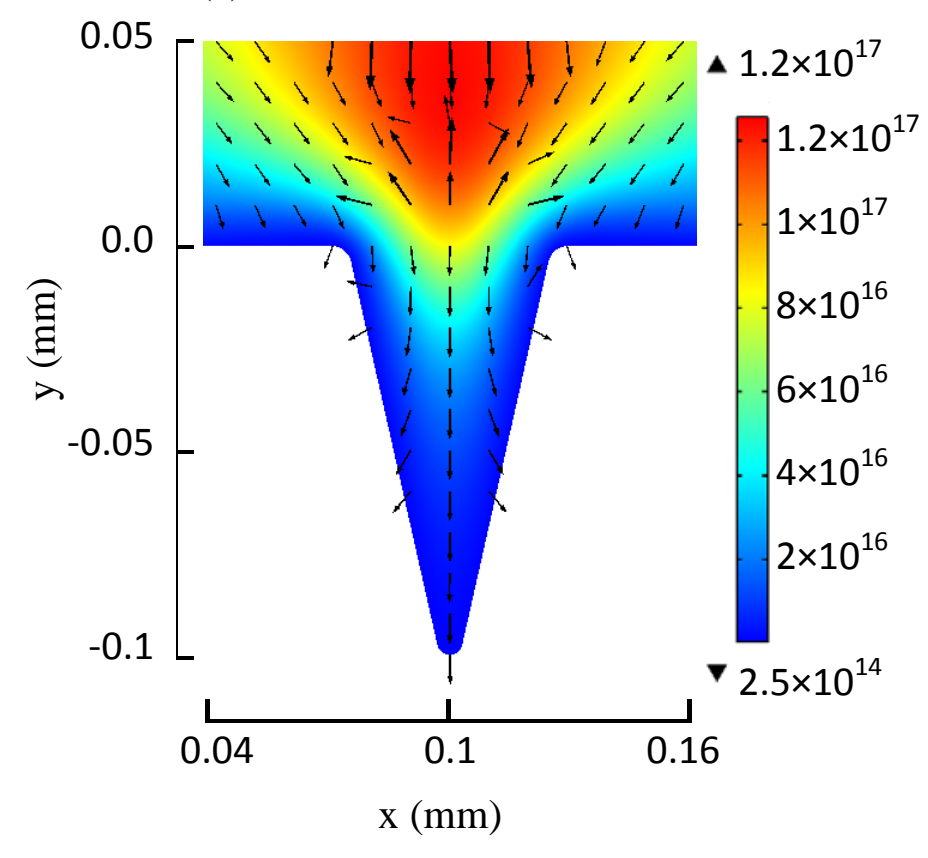

Figure 11 
(a)

1
2
3
4
5
6
7
8
9
10
11
12
13
14
15
16
17
18
19
20
21
22
23
24
25
26
27
28
29
30
31
32
33
34
35
36
37
38
39
40
41
42
43
44
45
46 (b)

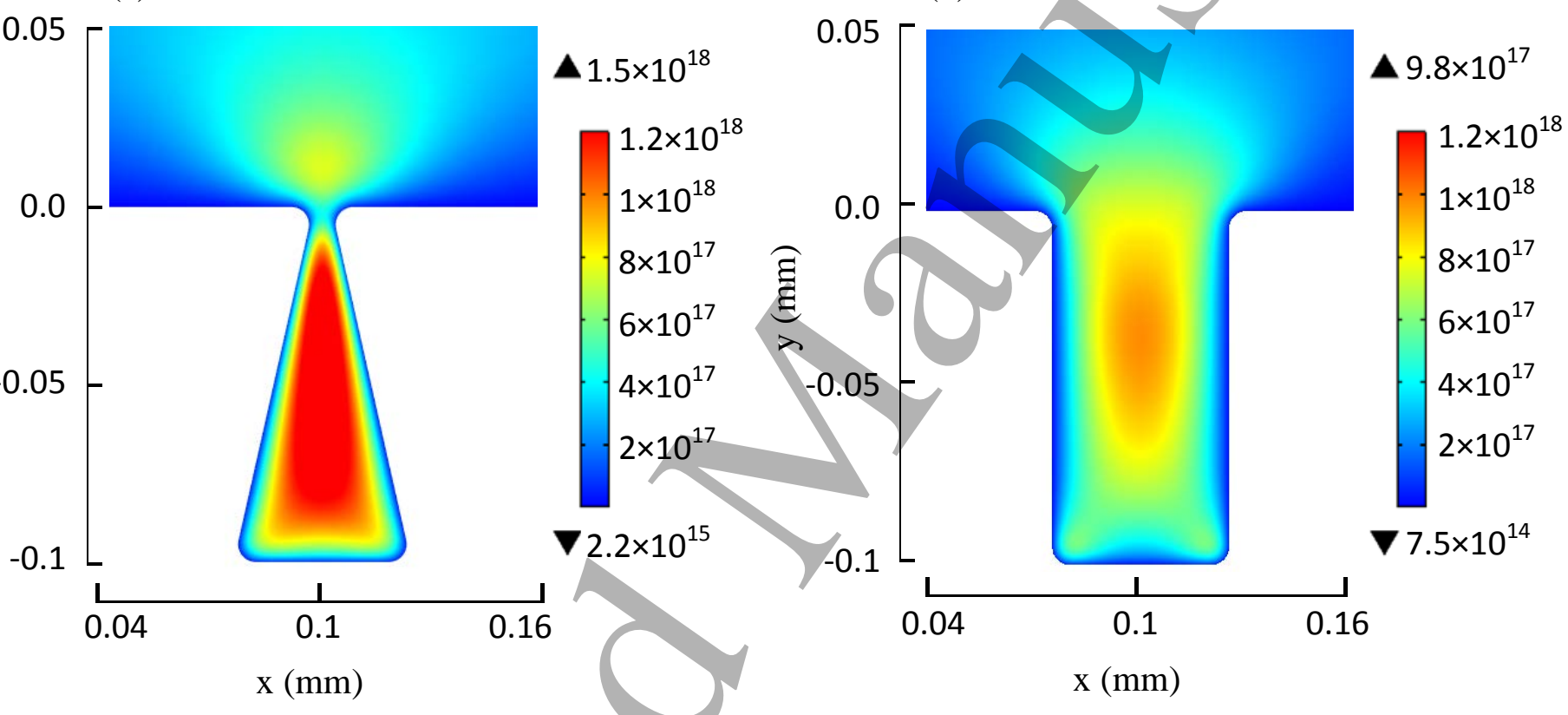

(c)

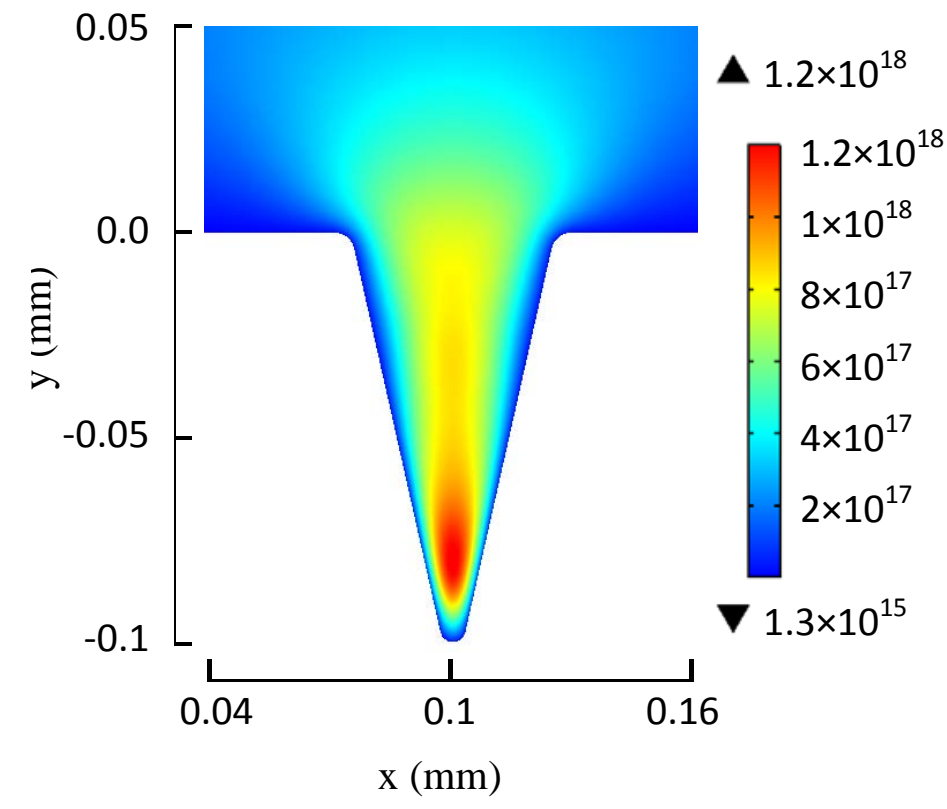

Figure 12 
(a)

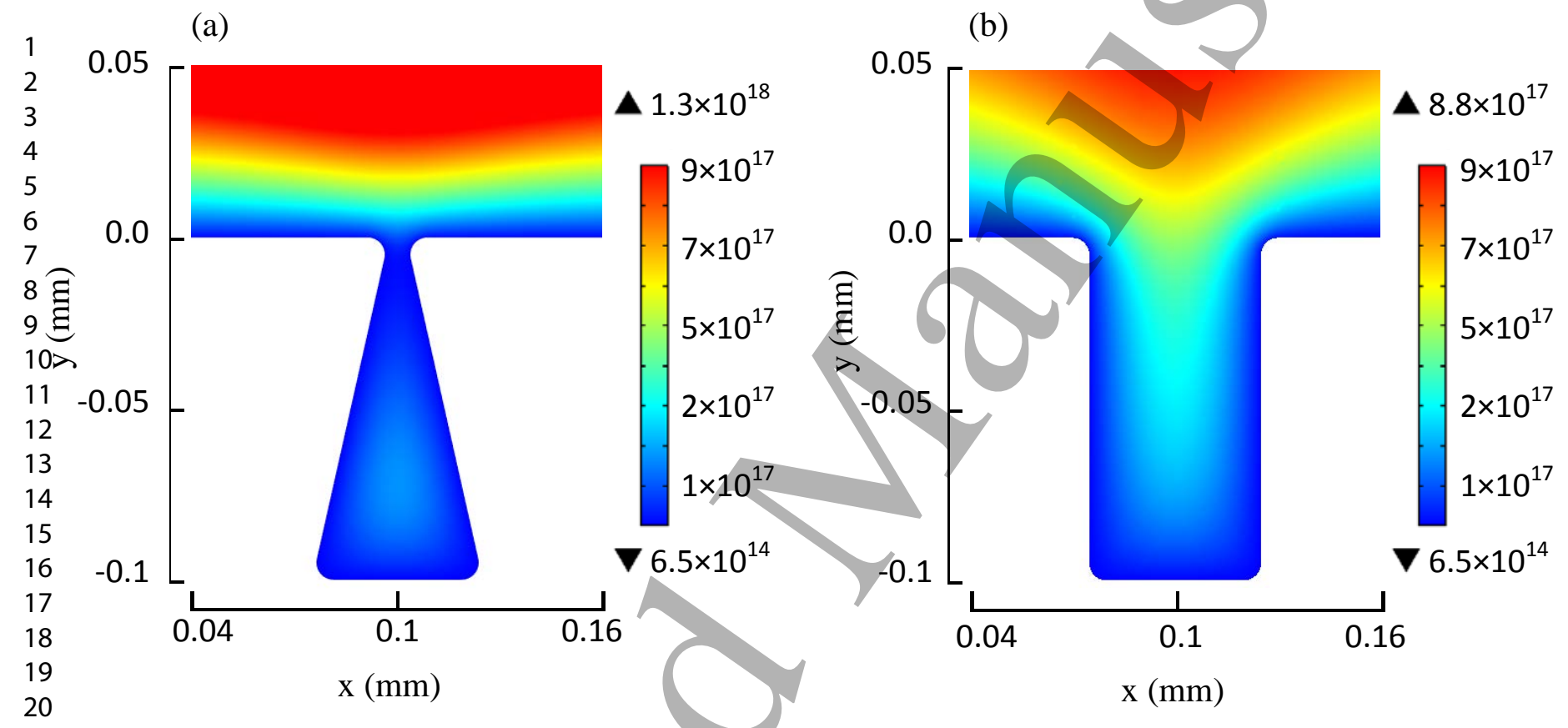

(c)

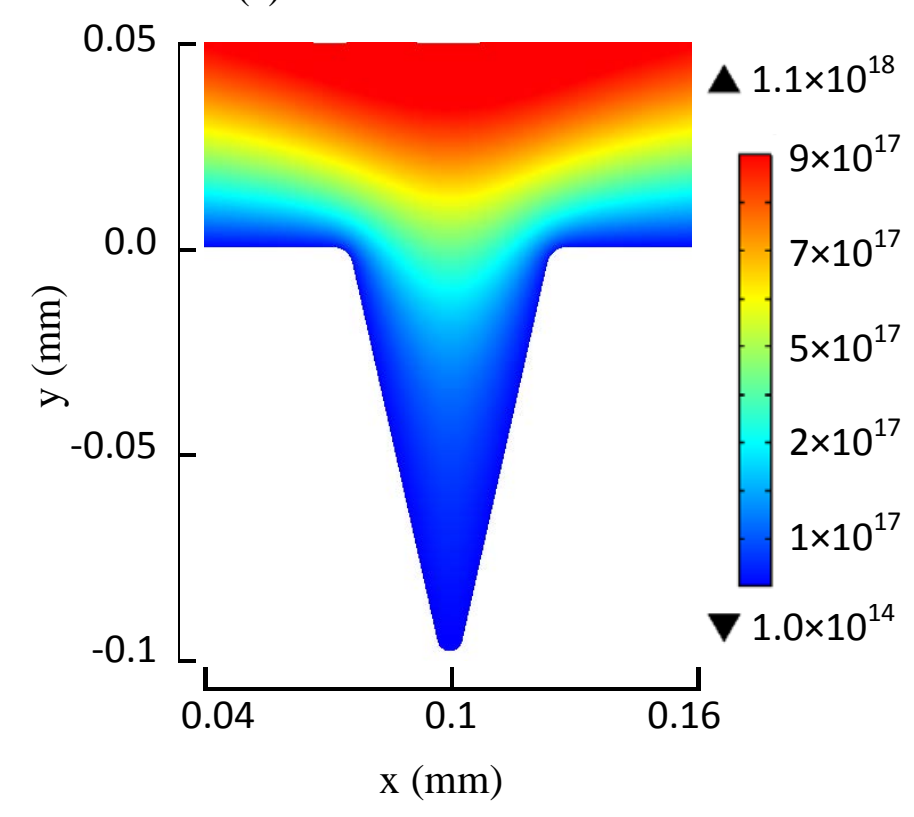

Figure 13 


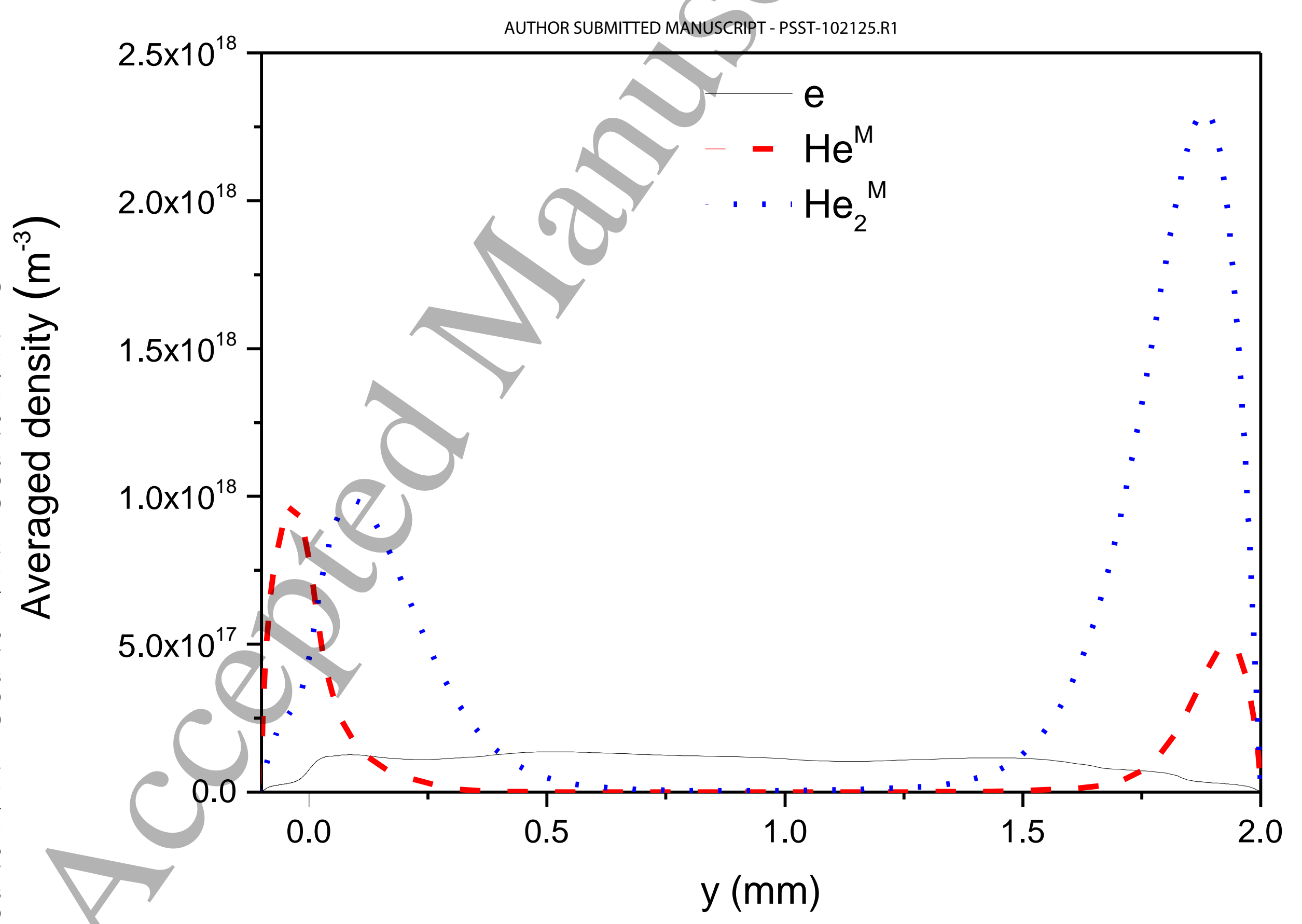

Page 36 of 37 


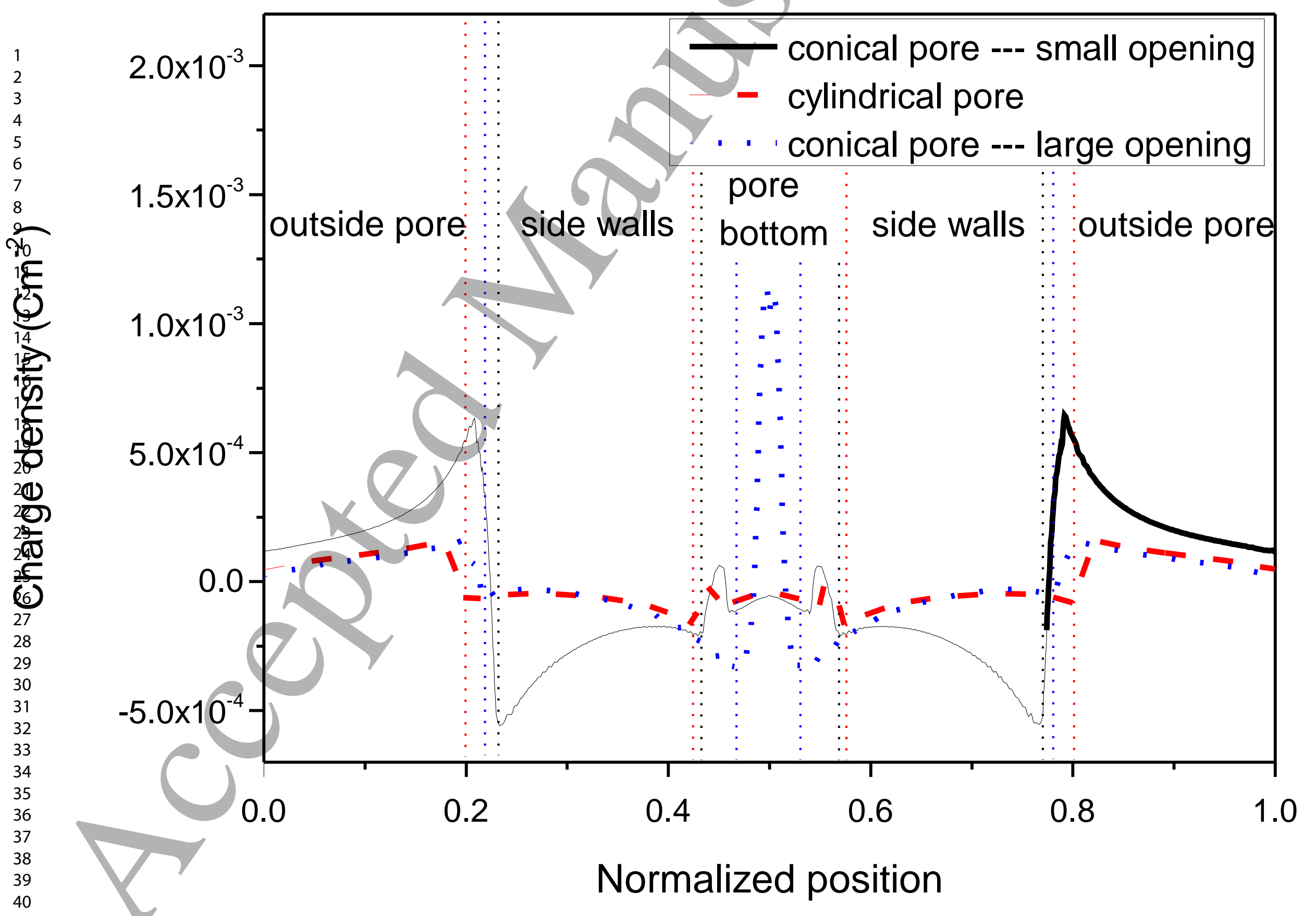

Figure 15 